\title{
Revealing the Origin of the Specificity of Calcium and Sodium Cations Binding to Adsorption Monolayers of Two Anionic Surfactants
}

\author{
Fatmegyul Mustan, ${ }^{\mathrm{a}}$ Anela Ivanova,${ }^{\mathrm{b}}$ Slavka Tcholakova, ${ }^{\mathrm{a}}$ Nikolai Denkov ${ }^{\mathrm{a}}$ \\ ${ }^{a}$ Department of Chemical and Pharmaceutical Engineering \\ ${ }^{b}$ Department of Physical Chemistry \\ Faculty of Chemistry and Pharmacy, \\ University of Sofia, 1 James Bourchier Ave., 1164 Sofia, Bulgaria \\ *Corresponding author; E-mail: aivanova@chem.uni-sofia.bg; Phone: ++35928161520; FAX \\ $++35929625438$
}

SUPPORTING INFORMATION 
Table S1. Content of the studied systems and dimensions of the simulation box at the two modelled surface concentrations, corresponding to area-per-molecule of $0.40 \mathrm{~nm}^{2}$ (concentrated) or $0.81 \mathrm{~nm}^{2}$ (diluted), respectively. The number of surfactant molecules and sodium ions (18 of each) is the same in all systems.

\begin{tabular}{|c|c|c|c|c|c|c|c|}
\hline System & $\mathbf{H}_{2} \mathbf{O}$ & $\mathrm{Ca}^{2+}$ & $\mathrm{Cl}^{-}$ & $x, \mathbf{n m}$ & $y, \mathrm{~nm}$ & $z, \mathbf{n m}$ & $\begin{array}{l}\text { Aqueous film } \\
\text { thickness, nm }\end{array}$ \\
\hline \multicolumn{8}{|c|}{ Concentrated layers } \\
\hline LAS & 391 & 0 & 0 & \multirow{11}{*}{1.9} & \multirow{11}{*}{1.9} & \multirow{11}{*}{17.0} & \multirow{11}{*}{4.43} \\
\hline SLES & 391 & 0 & 0 & & & & \\
\hline $\mathbf{L A S}+\mathrm{Ca}^{2+}$ & 364 & 9 & 18 & & & & \\
\hline $\mathrm{SLES}+\mathrm{Ca}^{2+}$ & 364 & 9 & 18 & & & & \\
\hline $\mathrm{LAS}+\mathrm{SLES}+\mathrm{Ca}^{2+}$ & 367 & 9 & 18 & & & & \\
\hline $\mathrm{C}_{16} \mathrm{SO}_{3}+\mathrm{SLES}+\mathrm{Ca}^{2+}$ & 390 & 9 & 18 & & & & \\
\hline $\mathrm{LAS12}+\mathrm{C}_{12} \mathrm{EOSO}_{3}+\mathrm{Ca}^{2+}$ & 366 & 9 & 18 & & & & \\
\hline $\mathrm{LAS12}+\mathrm{SDS}+\mathrm{Ca}^{2+}$ & 393 & 9 & 18 & & & & \\
\hline LAS12 + SLES + $\mathrm{Ca}^{2+}$ & 403 & 9 & 18 & & & & \\
\hline $\mathrm{SLES}+\mathrm{SDS}+\mathrm{Ca}^{2+}$ & 384 & 9 & 18 & & & & \\
\hline $\mathrm{SDS}+\mathrm{C}_{12} \mathrm{SO}_{3}+\mathrm{Ca}^{2+}$ & 415 & 9 & 18 & & & & \\
\hline \multicolumn{8}{|c|}{ Diluted layers } \\
\hline LAS & 861 & 0 & 0 & \multirow{5}{*}{2.7} & \multirow{5}{*}{2.7} & \multirow{5}{*}{17.0} & 3.99 \\
\hline SLES & 861 & 0 & 0 & & & & 4.10 \\
\hline $\mathbf{L A S}+\mathrm{Ca}^{2+}$ & 834 & 9 & 18 & & & & 3.99 \\
\hline $\mathrm{SLES}+\mathrm{Ca}^{2+}$ & 834 & 9 & 18 & & & & 4.10 \\
\hline $\mathrm{LAS}+\mathrm{SLES}+\mathrm{Ca}^{2+}$ & 858 & 9 & 18 & & & & 4.20 \\
\hline LAS12B6 + SLES + $\mathrm{Ca}^{2+}$ & 1072 & 9 & 18 & 2.9 & 2.9 & 17 & 4.43 \\
\hline
\end{tabular}



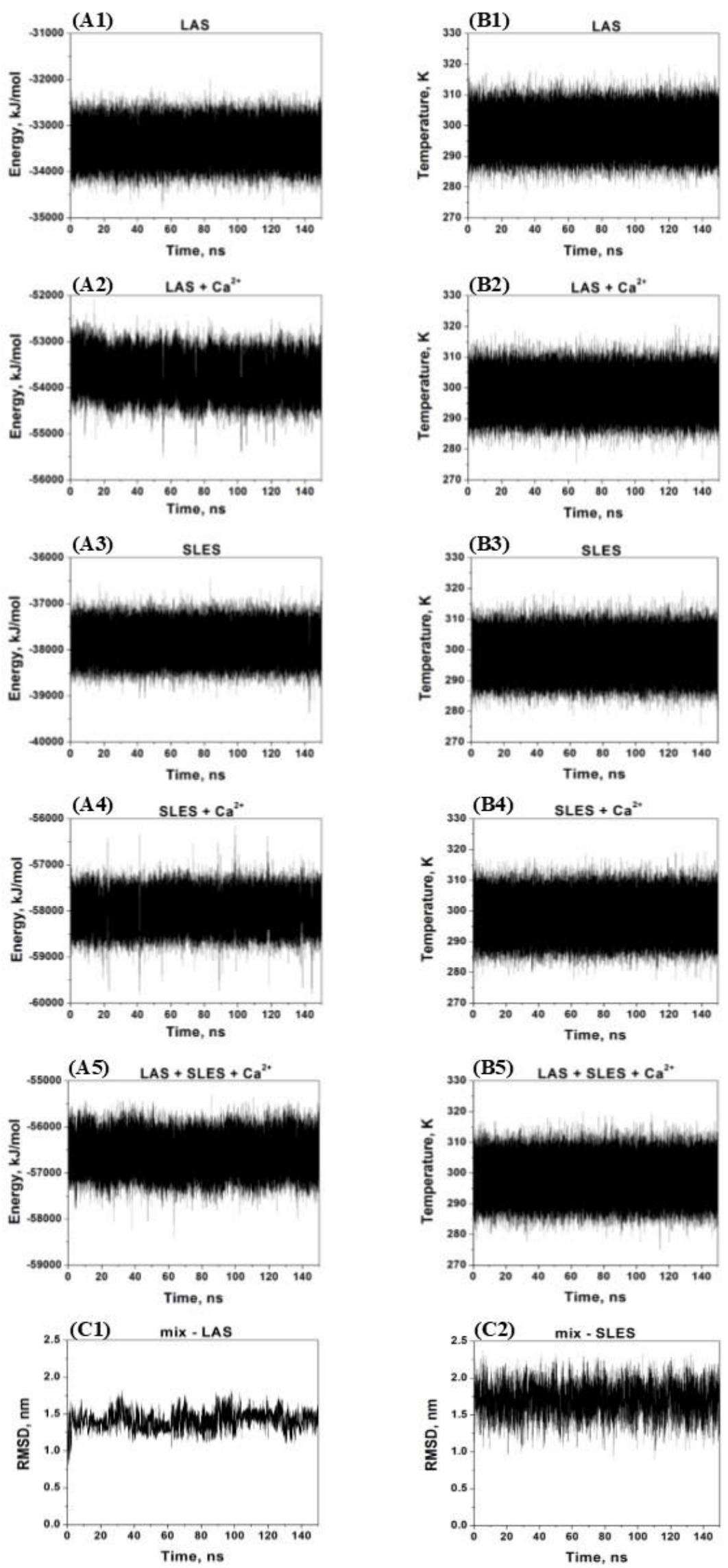

Figure S1. (A) Total energy and (B) total temperature as functions of the simulation time for all studied systems with low surface concentration: (1) LAS, (2) LAS+Ca ${ }^{2+}$, (3) SLES, (4) SLES+Ca ${ }^{2+}$, (5) LAS+SLES+Ca ${ }^{2+}$. (C) RMSD of the surfactants: (1) LAS and (2) SLES, as a function of simulation time is shown to illustrate a typical profile in the asymmetric system. 

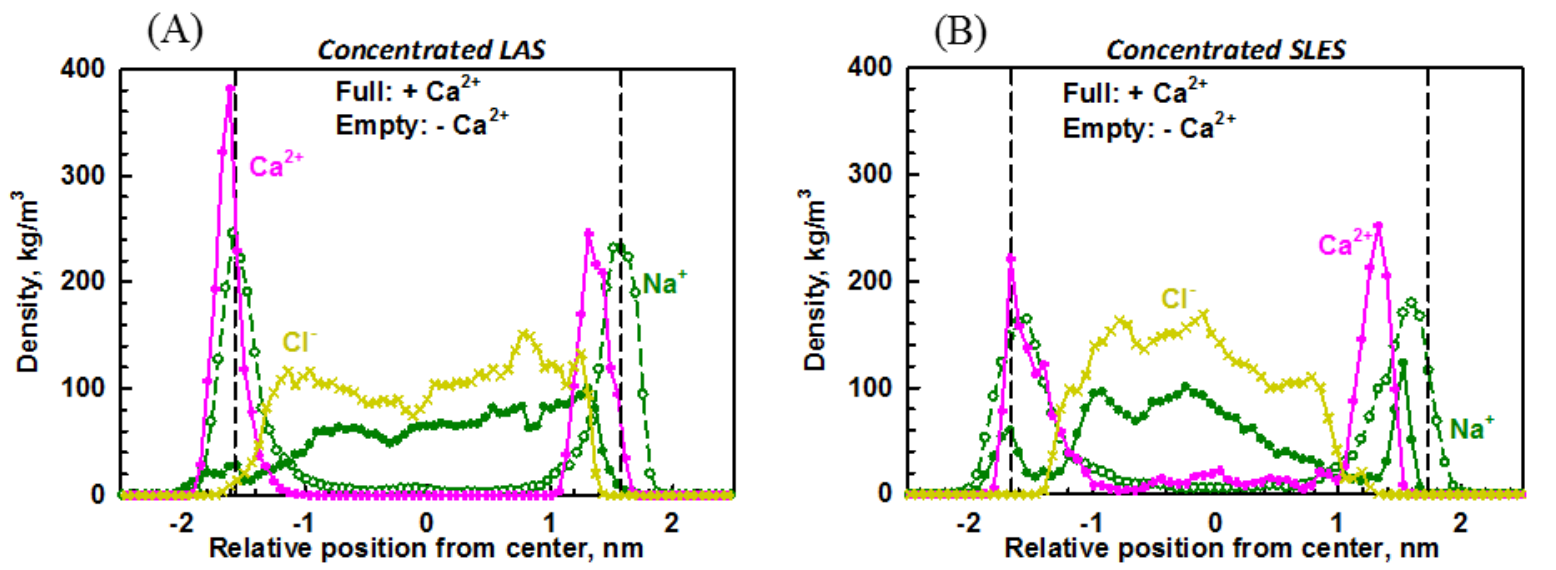

Figure S2. Density profiles of the inorganic ions in pure (A) LAS and (B) SLES concentrated systems - green circles for $\mathrm{Na}^{+}$(full: in the presence of $\mathrm{Ca}^{2+}$; empty: in the absence of $\mathrm{Ca}^{2+}$ ), pink crosses for $\mathrm{Ca}^{2+}$, yellow $\mathrm{X}$ for $\mathrm{Cl}^{-}$. The black dashed lines indicate the position of the surface corresponding to water density of $500 \mathrm{~kg} / \mathrm{m}^{3}$.
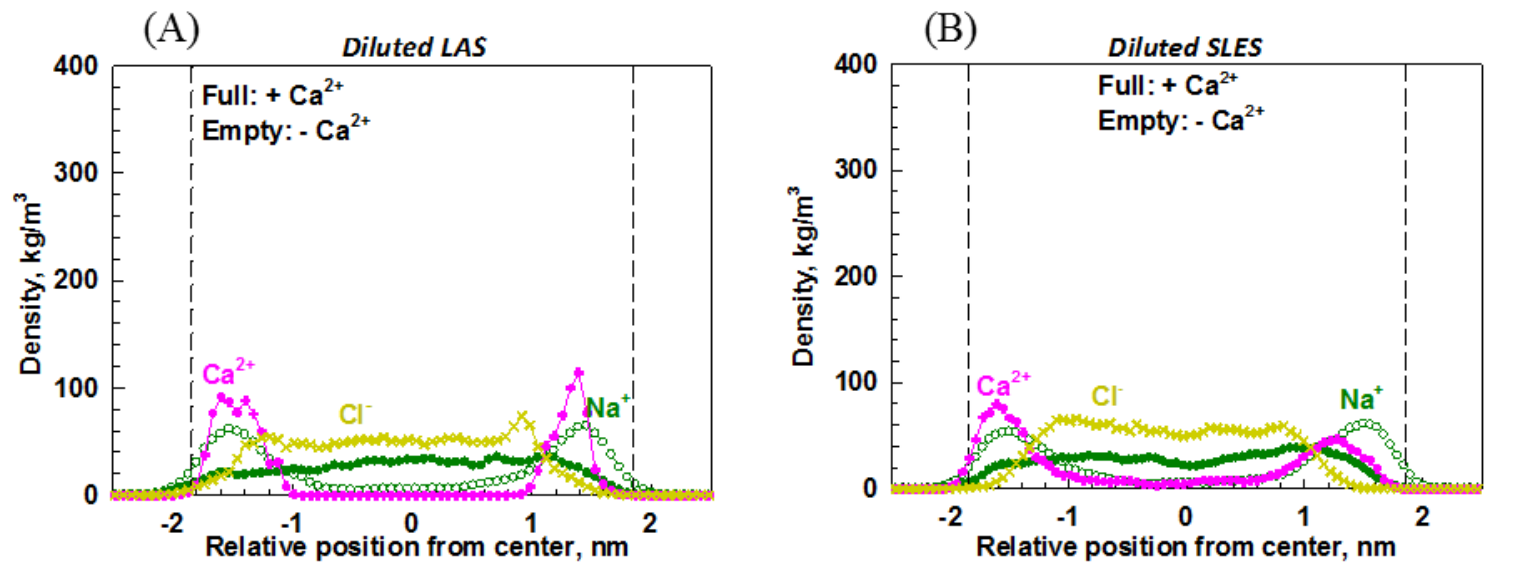

Figure S3. Density profiles of the inorganic ions $\left(\mathrm{Na}^{+}\right.$: green circles, $\mathrm{Ca}^{2+}$ : pink crosses, $\mathrm{Cl}^{-}$: yellow $\times$ ) in pure (A) LAS and (B) SLES systems in the presence (full symbols) and in the absence (empty symbols) of $\mathrm{Ca}^{2+}$ with dilute adsorption monolayers. 

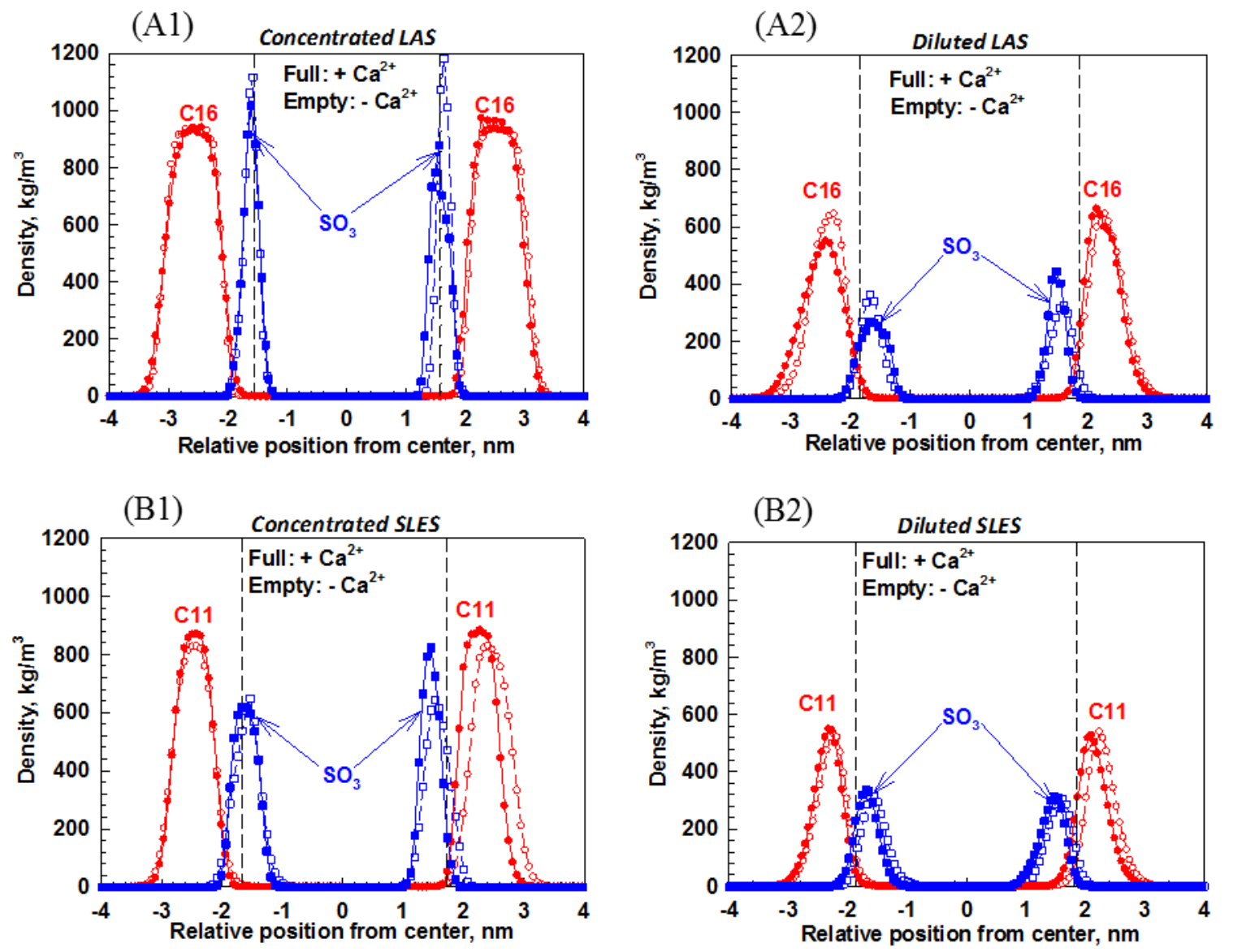

Figure S4. Density profiles of the hydrophobic alkyl chains (red circles) and $-\mathrm{SO}_{3}$ groups (blue squares) in pure (A) LAS and (B) SLES monolayers in the presence (solid line and full symbols) and in the absence (dashed line and empty symbols) of $\mathrm{Ca}^{2+}$ for (1) concentrated and (2) diluted monolayers.

The data on Figure S4 show that the hydrophobic tails of the molecules are entirely immersed in the vacuum phase and the sulfate and sulfonate groups are very close to the water interface in the systems with concentrated surface layers. The sulfate group of the SLES molecules is slightly more immersed in water than the sulfonate group in the LAS molecules. The reason for this behavior is the difference in the fragments between the hydrophobic and the hydrophilic parts of the two molecules. In LAS this fragment is a hydrophobic benzene ring while in SLES it is an ethoxy group, which is more hydrophilic. When the surfaces are diluted, the hydrophilic heads of both surfactants are entirely immersed in the water phase, even a small fraction of the alkyl chain is also hydrated. This is an expected behavior due to the steric effects which change the molecular packing. The position of the surfactant molecules with respect to the interface is not influenced markedly by the presence of $\mathrm{Ca}^{2+}$. 

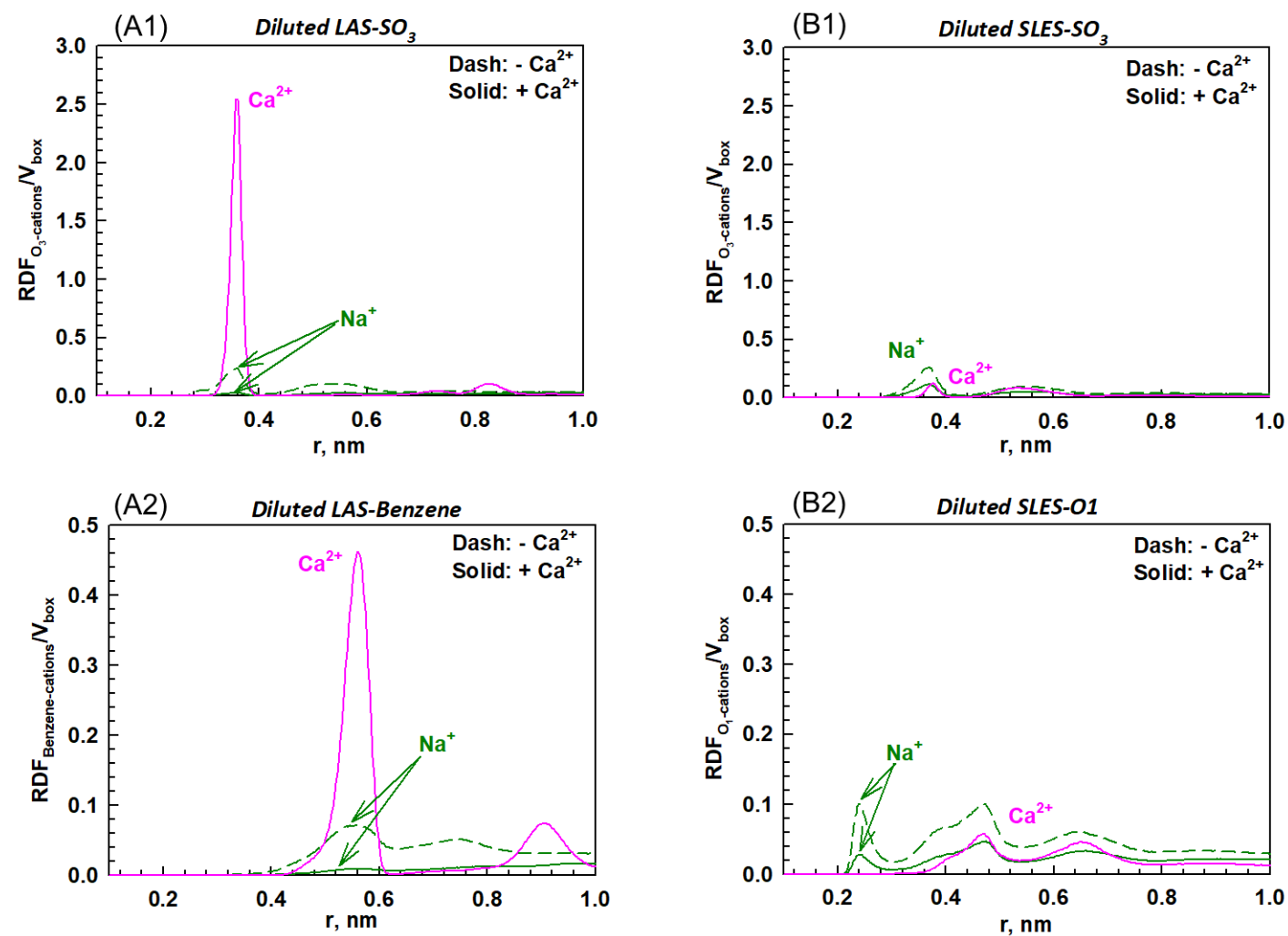

Figure S5. RDFs between the cations (green lines $\mathrm{Na}^{+}$, pink lines $\mathrm{Ca}^{2+}$ ) and the center-ofmass of (1) $-\mathrm{SO}_{3}{ }^{-}$groups and (2) benzene ring/atom $\mathrm{O} 1$ (denoted in Figure 1) of (A) LAS and (B) SLES, respectively, in the diluted systems in the presence (solid lines) and in the absence (dashed lines) of $\mathrm{Ca}^{2+}$.

In respect to the RDFs presented on Figure S5, in the LAS models, prominent peaks with the same intensity at a distance of $0.56 \mathrm{~nm}$, corresponding to interaction between the calcium ion and the benzene ring, are observed with both monolayers, concentrated and diluted. In the absence of $\mathrm{Ca}^{2+}$, there is again a peak in the RDF between the $\mathrm{Na}^{+}$ion and the aromatic ring but its intensity and position significantly depend on the surface concentration. Milder interactions between the $\mathrm{Na}^{+}$ions and the phenyl fragment of LAS than those with the calcium ions are obtained.

In the pure SLES systems, the intensity of the RDF peak for the strongest interaction between $\mathrm{Ca}^{2+}$ and the $-\mathrm{SO}_{3}$ residue is lower when compared to that between $\mathrm{Na}^{+}$and $-\mathrm{SO}_{3}$ when calcium is absent from the system. A second peak of the RDF between $\mathrm{Na}^{+}$and the outer sulfate oxygens of SLES with comparable intensity to that of the first one is obtained at a distance of $0.46 \mathrm{~nm}$. This distance coincides with the distance where the first peak of the RDF between $\mathrm{Ca}^{2+}$ and the linking oxygen is located. Again, the peak intensities to the $\mathrm{Na}^{+}$ ions are higher than those to $\mathrm{Ca}^{2+}$ as was in the case of the charged oxygen in SLES. Very low peaks at $0.65 \mathrm{~nm}$ are seen for both cations, which is most probably due to the mobility of the ions along the $\mathrm{z}$ coordinate during the simulations. 
(A1) LAS
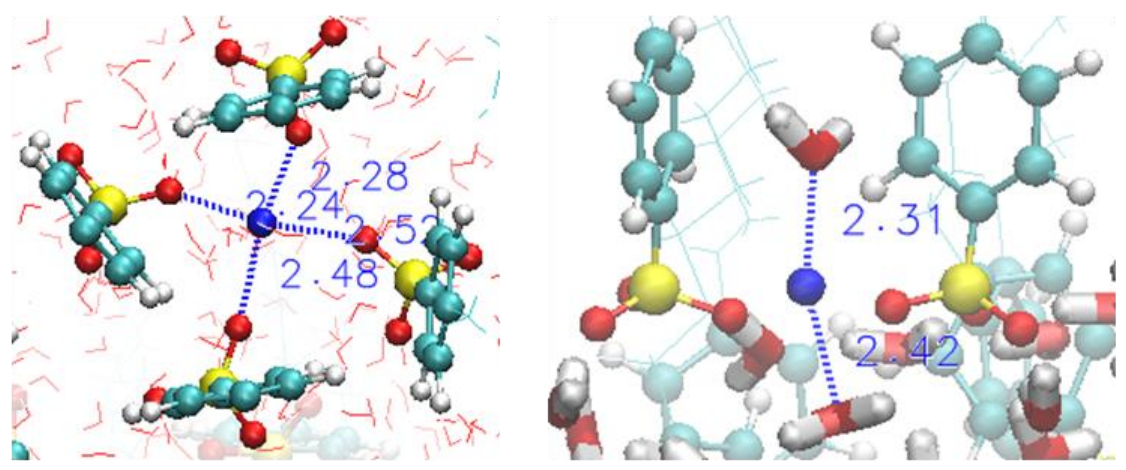

(A2) $\mathrm{LAS}+\mathrm{Ca}^{2+}$
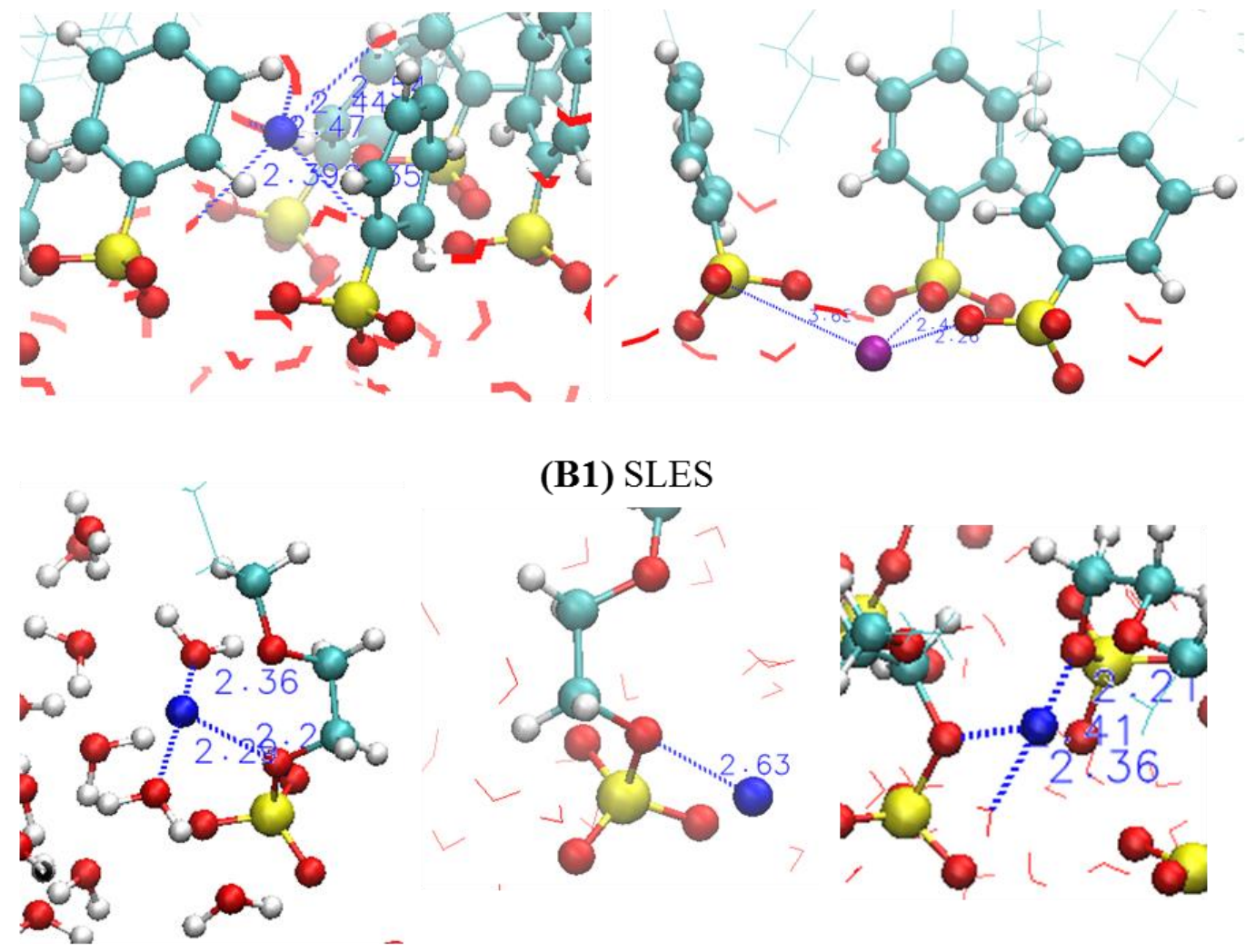

(B1) SLES

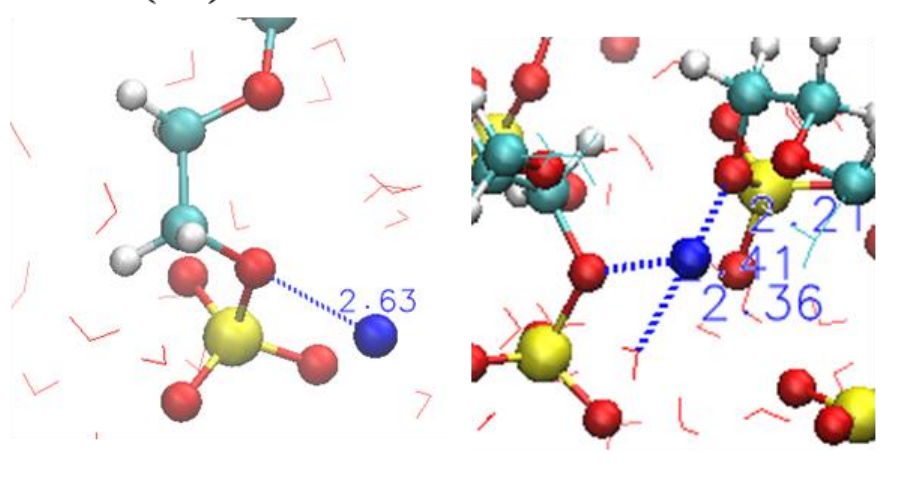

(B2) $\mathrm{SLES}+\mathrm{Ca}^{2+}$
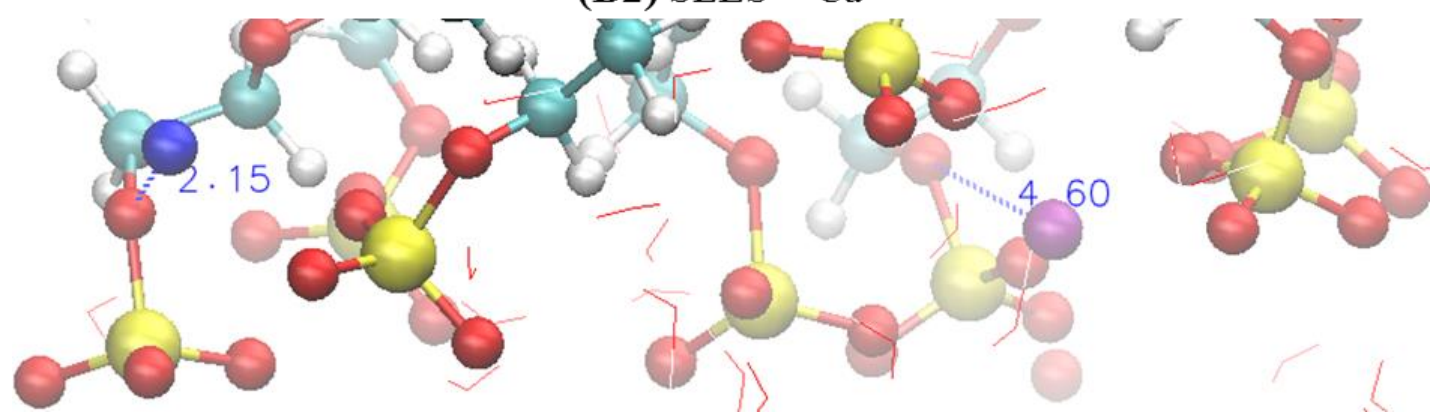

Figure S6. Illustration of $\mathrm{Na}^{+}$cation (blue balls) and $\mathrm{Ca}^{2+}$ (purple balls) in the vicinity of the surfactants heads ( $\mathrm{O}$ atoms: red balls, $\mathrm{S}$ atoms: yellow balls) with reduced hydration shell $\left(\mathrm{H}_{2} \mathrm{O}\right.$ : red lines) in the monolayers of (A) LAS and (B) SLES in the concentrated singlesurfactant systems (1) in the absence and (2) in the presence of $\mathrm{Ca}^{2+}$. The shown distances are in $\AA$. 
For the $\mathrm{Na}^{+}$ion to enter between the surfactant molecules it has to keep only one or two water molecules in the hydration shell, which is obviously not preferable for this cation. Nevertheless, this penetration occurs, as seen in the visual inspection of the trajectories, but for very short time of the simulations, $<1 \mathrm{~ns}$ (Figure S6). Hence, the complete removal of the hydration shell is energetically painful for $\mathrm{Na}^{+}$and that is why the average number of water molecules in the hydration shell is in the range of 4 to 5 , in the last $50 \mathrm{~ns}$ of the trajectories.

The $\mathrm{Ca}^{2+}$ ion is prone to retaining similar number of water molecules in its hydration shell in all environments which does not allow it to penetrate among the surfactant molecules. In SLES monolayers $\mathrm{Ca}^{2+}$ keeps almost all surrounding water molecules, ca. 7 water molecules remain in its hydration shell. When it is adsorbed on LAS layers, $\mathrm{Ca}^{2+}$ strips off half of its hydration shell but still does not enter in-between the surfactants heads (Figure S6).

(A) $\mathbf{L A S}+\mathrm{Ca}^{2+}$

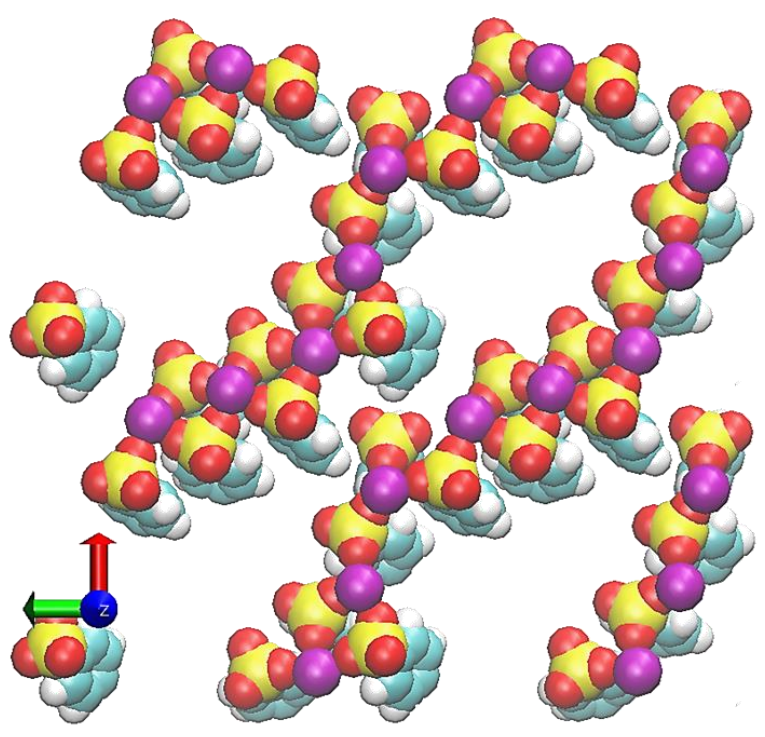

(B) SLES $+\mathrm{Ca}^{2+}$

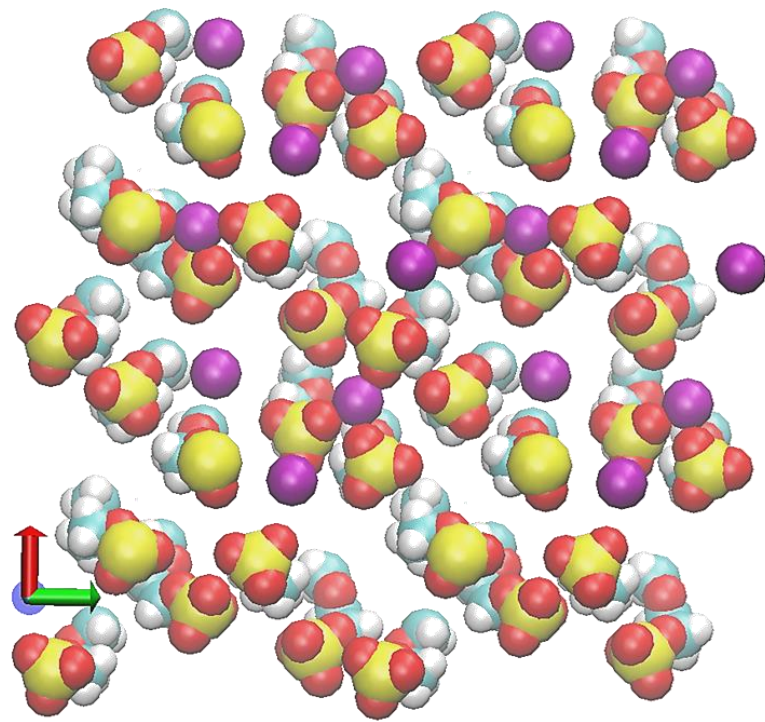

Figure S7. Illustration of the monolayers in the pure systems with adsorbed $\mathrm{Ca}^{2+}$ (purple balls) between $\mathrm{O}$ atoms (red balls) of (A) LAS and (B) SLES. The yellow, white and cyan balls are $\mathrm{S}, \mathrm{H}$ and $\mathrm{C}$ atoms, respectively. 
(A) Diluted

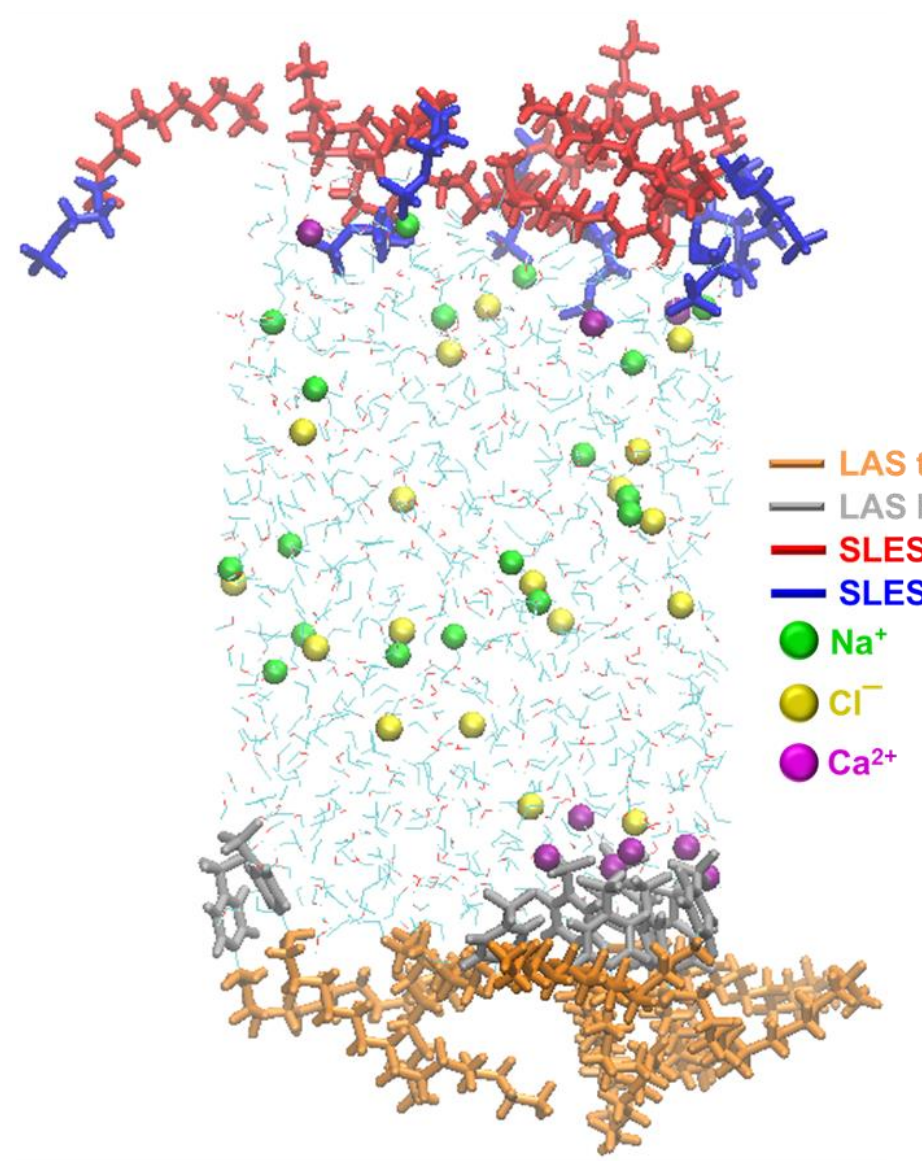

(B) Concentrated

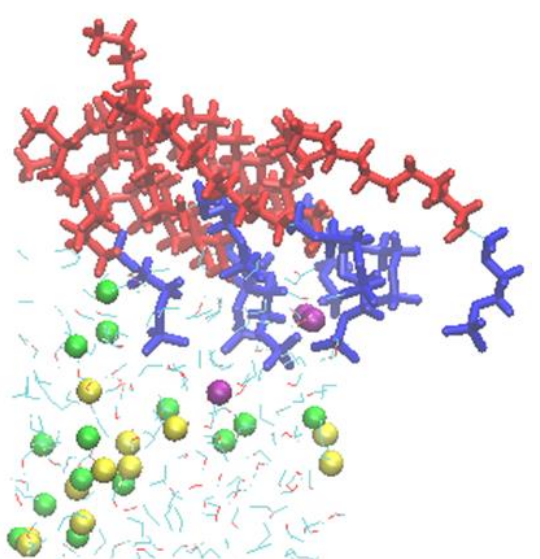

Figure S8. Snapshots of the asymmetric (A) diluted and (B) concentrated LAS and SLES systems after $150 \mathrm{~ns}$ of simulation. 

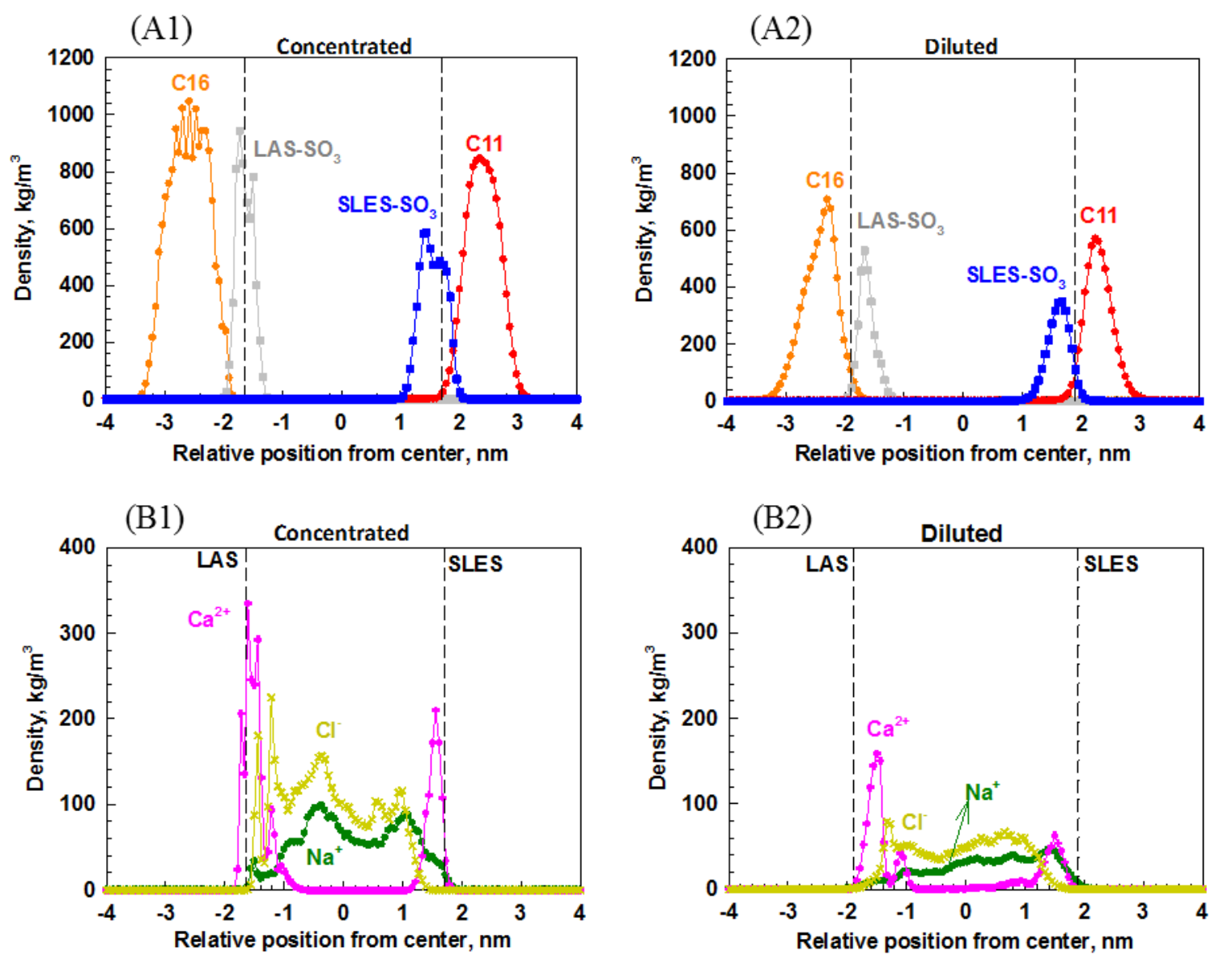

Figure S9. Mass density profiles normal to the interface of (A) key atoms from the hydrocarbon chains and of the - $\mathrm{SO}_{3}$ groups in LAS and SLES and (B) of the inorganic ions green circles for $\mathrm{Na}^{+}$, pink crosses for $\mathrm{Ca}^{2+}$, and yellow $\mathrm{X}$ for $\mathrm{Cl}^{-}$, in (1) concentrated and (2) diluted systems.

The behavior of the surfactants with respect to their positions at the vacuum-water interface is similar to that in the single-surfactant systems. In the concentrated monolayers the alkyl chains are entirely in the gas phase and the polar groups are right at the interface. When the layer is diluted, the molecules are slightly more immersed in the water phase (Figure S9 (A1,A2)). Concerning the density profiles of the ions, again all calcium cations are adsorbed on the surfaces, while the sodium and chloride ions are dispersed in the bulk (Figure S9 (B1, B2)). The $\mathrm{Ca}^{2+}$ density is higher at the LAS monolayer than at the SLES one. This result confirms the higher affinity of $\mathrm{Ca}^{2+}$ to LAS. The effect is illustrated by the z-coordinate of the calcium ions as a function of the simulation time (see Figure S10). 

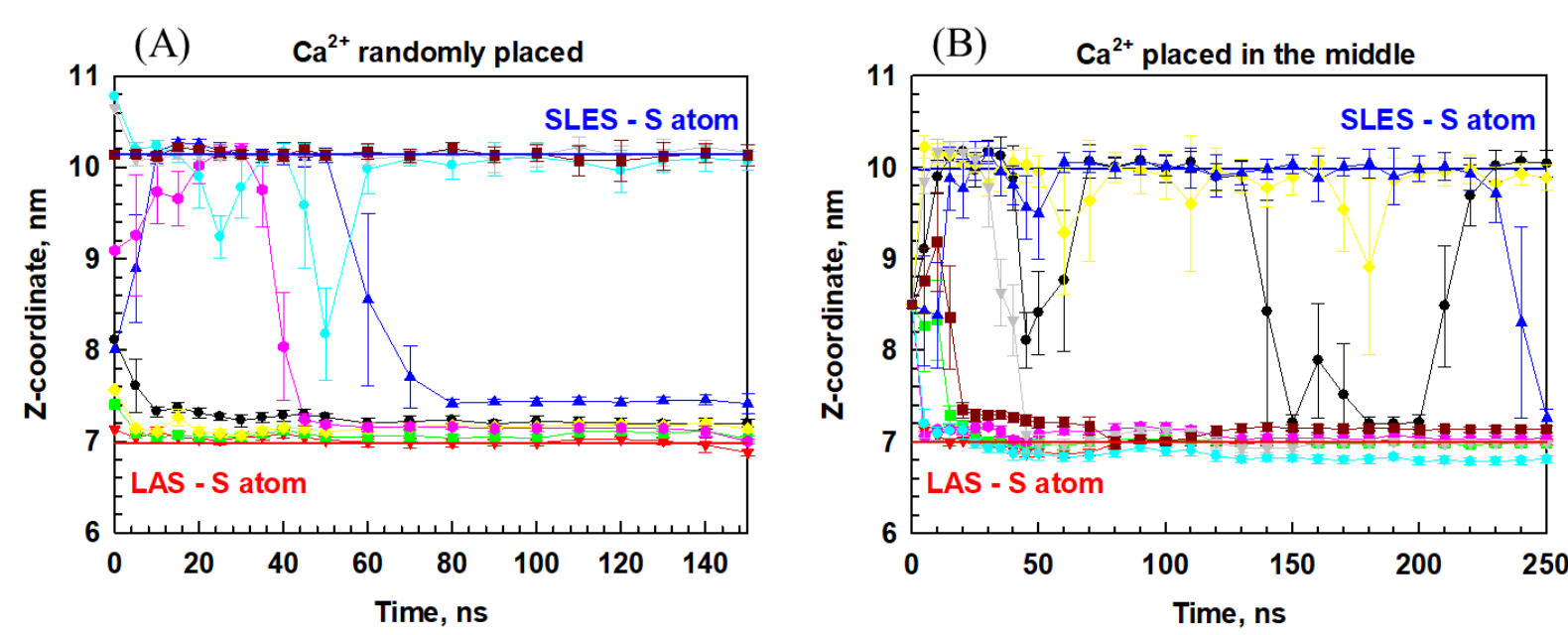

Figure S10. Z-coordinates of all $\mathrm{Ca}^{2+}$ ions as functions of time in the concentrated asymmetric systems when the $\mathrm{Ca}^{2+}$ ions are initially (A) randomly placed in the box or (B) placed in the middle of the box at the same distance to both surfaces.

The preference of $\mathrm{Ca}^{2+}$ for adsorption to LAS monolayers is confirmed by the averaged $z$-coordinates of the cations in the last $50 \mathrm{~ns}$ of the simulations with two different starting positions (Figure S10) which are used to evaluate the mobility of the counterions in the simulation box. The $z$-coordinates of the counterions and the sulfate/sulfonate $S$-atoms in the surfactant molecules are estimated for the whole simulation time. These coordinates, as a function of simulation time, for the systems with different initial positions of $\mathrm{Ca}^{2+}$ ions demonstrate very clearly that the calcium ions diffuse initially between the two surfaces but after $60 \mathrm{~ns}$ of the simulations all of them are fixed at one of the two monolayers. 

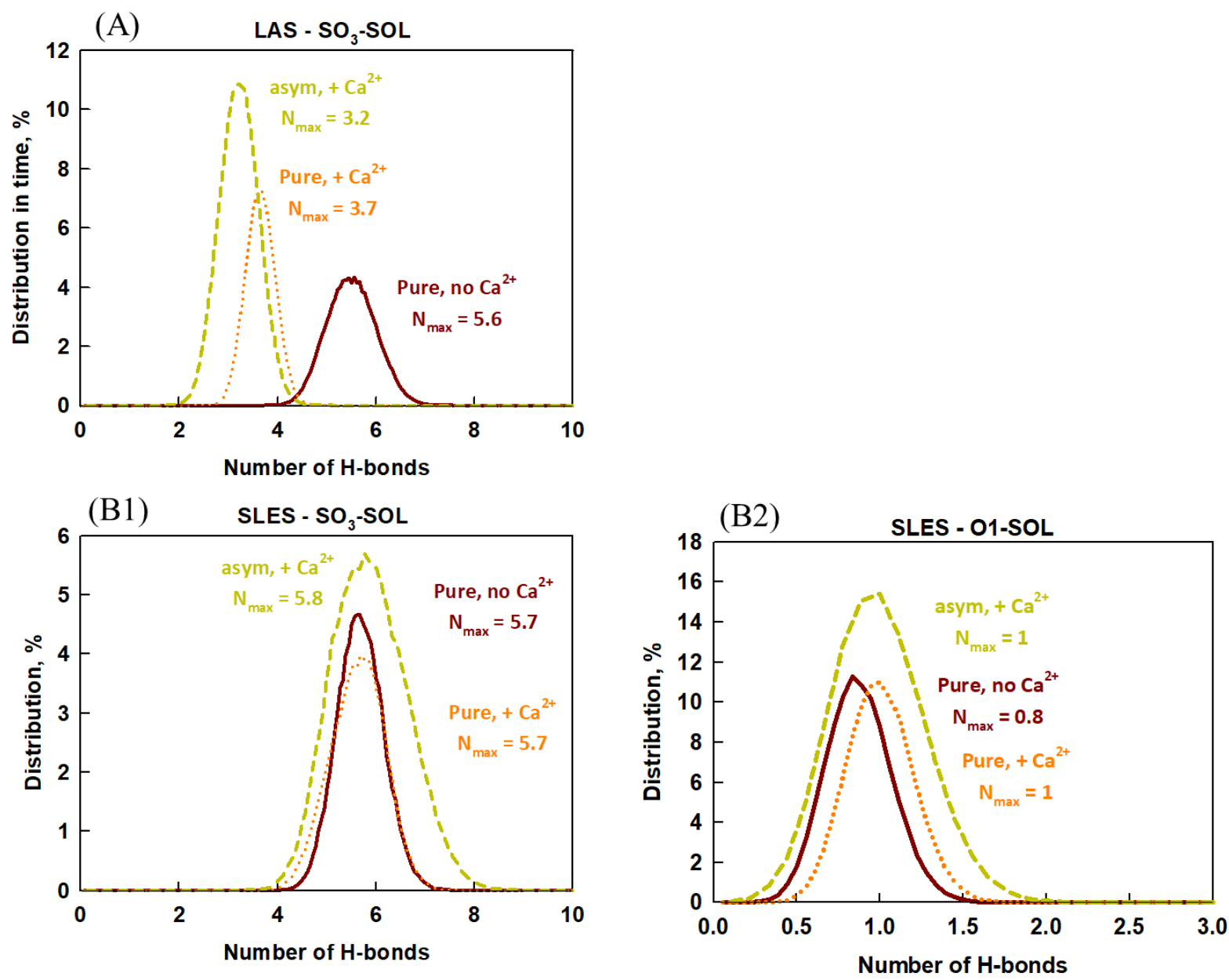

Figure S11. Distribution of the number of hydrogen bonds formed between the $-\mathrm{SO}_{3}$ group and the water molecules obtained from the last $50 \mathrm{~ns}$ of the MD trajectories for (A1) LAS and (B1) SLES, and (B2) between the inner O1 atom in SLES and water. There are no H-bonds between the benzene ring of LAS and the water molecules. 


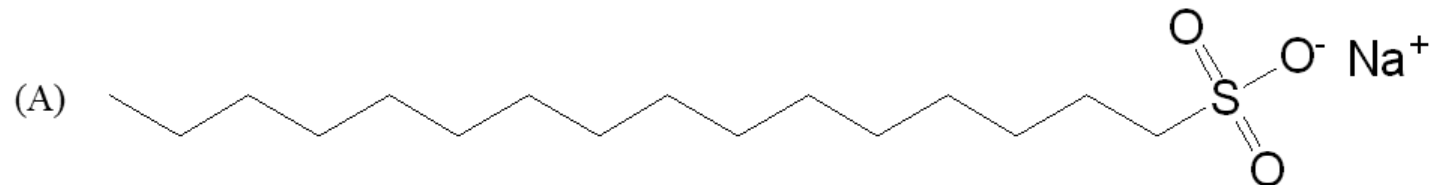

(B)<smiles>CCCCCCCCCCCCc1ccc(S(=O)(=O)[O-])cc1</smiles>

(C)<smiles>CCCCCCCCCCCCOCCS(=O)(=O)[NH2+][Na]</smiles>

(D)<smiles>CCCCCCCCCCCCOS(=O)(=O)[O-]</smiles>

(E)<smiles>CCCCCCCCCCCCS(=O)(=O)[O-]</smiles>

(F)<smiles>CCCCCCC(CCCCC)c1ccc(S(=O)(=O)[O-])cc1</smiles>

Figure S12. Chemical structures of the molecules used as model surfactants: (A) sodium hexadecyl sulfonate, $\mathrm{C}_{16} \mathrm{SO}_{3}$, (B) dodecyl benzene sulfonate, LAS12, (C) dodecyl ethoxy sulfonate, $\mathrm{C}_{12} \mathrm{EOSO}_{3}$, (D) dodecyl sulfate, SDS, (E) dodecyl sulfonate, $\mathrm{C}_{12} \mathrm{SO}_{3}$, and (F) dodecyl 6-benzene sulfonate (LAS12B6). 


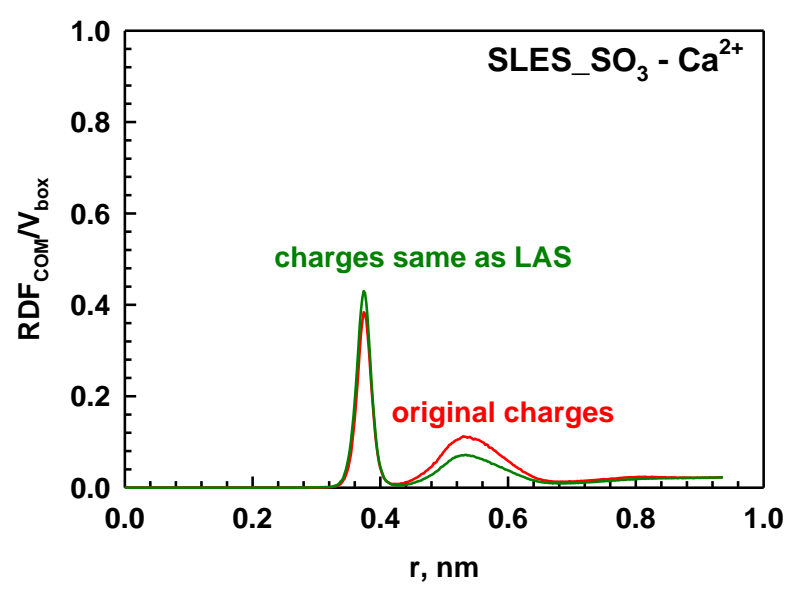

Figure S13. RDFs between the SLES charged heads and $\mathrm{Ca}^{2+}$ in the asymmetric systems of LAS/SLES with the original charges of SLES (red line) and with same charges of LAS and SLES (green line).
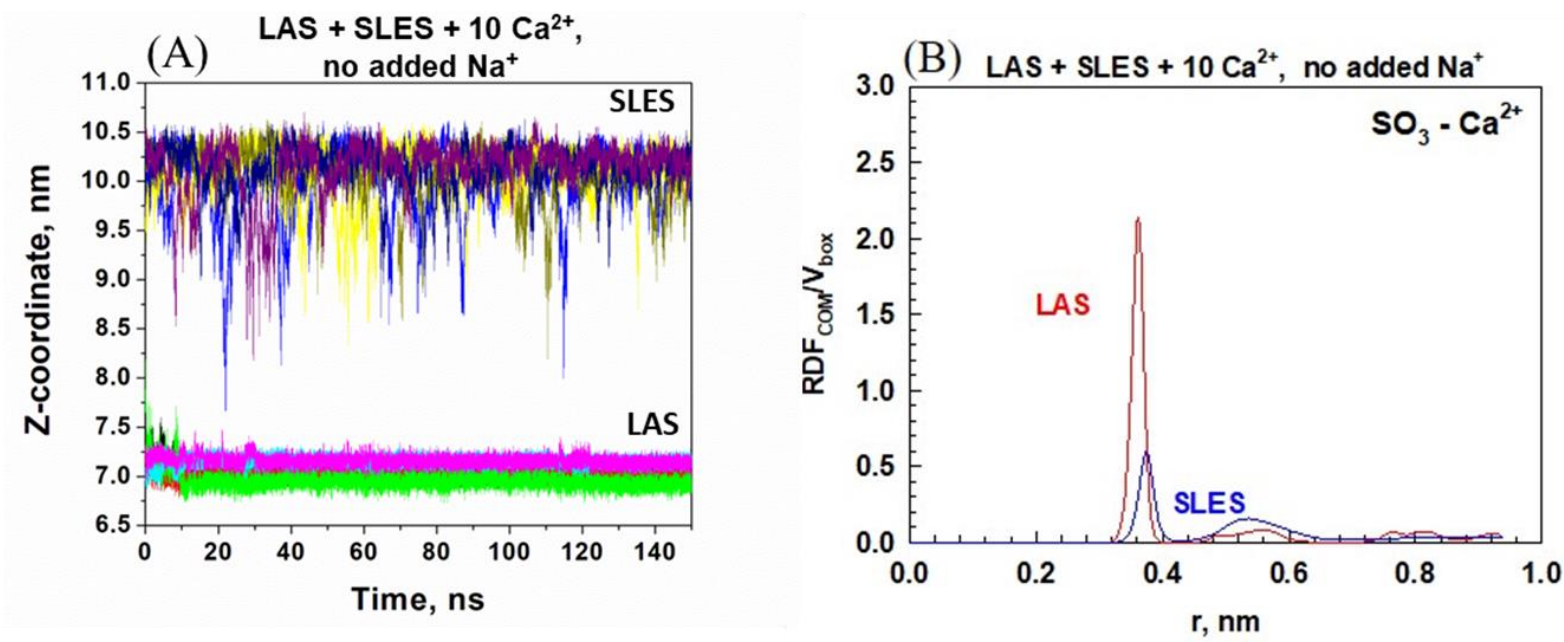

Figure S14. (A) $z$-coordinates of all $\mathrm{Ca}^{2+}$ ions vs. time in the model system without added $\mathrm{Na}^{+}$and (B) RDFs between the surfactant $\mathrm{SO}_{3}$ groups and $\mathrm{Ca}^{2+}$. 

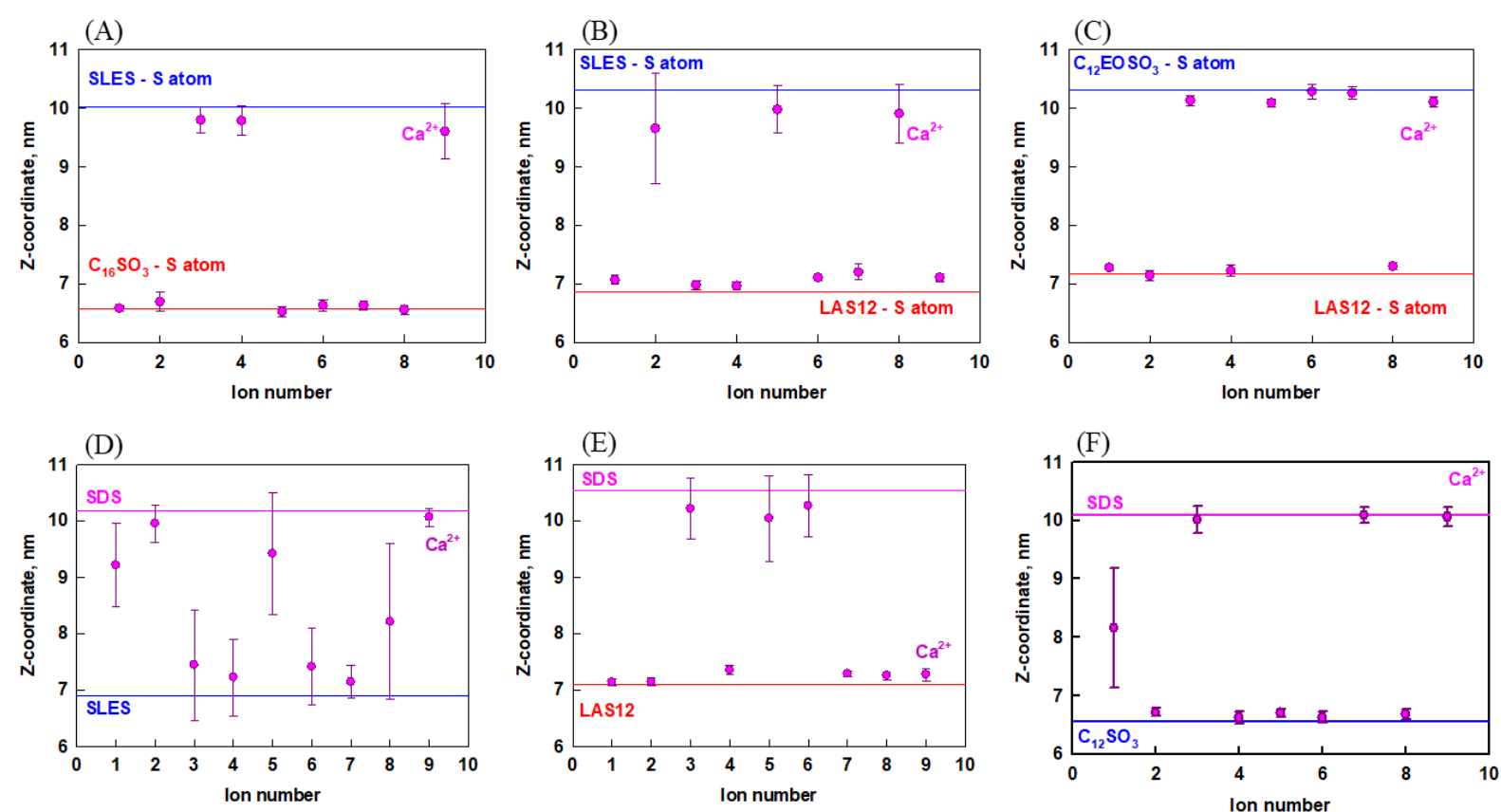

Figure S15. Average z-coordinates (with standard deviations) of all calcium ions in the last $50 \mathrm{~ns}$ of the simulations, relative to the mean coordinates of the sulfur atoms in the respective surfactants (LAS-like: red line, SLES-like: blue line, SDS: pink line) in the asymmetric systems: (A) $\mathrm{C}_{16} \mathrm{SO}_{3}+\mathrm{SLES}$, (B) LAS12 + SLES, (C) LAS12 + $\mathrm{C}_{12} \mathrm{EOSO}_{3}$, (D) SLES + SDS, (E) LAS12 + SDS, (F) $\mathrm{C}_{12} \mathrm{SO}_{3}+\mathrm{SDS}$.

To interpret the obtained results quantitatively, the following analysis is conducted for all model systems: $\mathrm{Ca}^{2+}$ average $z$-coordinates (Figure S15), RDFs between charged surfactant groups and $\mathrm{Ca}^{2+}$ (Figure 9 and Figure S16), hydration shells of the counterion, interaction energies between $\mathrm{Ca}^{2+}$ and the surfactant heads, and number of hydrogen bonds between $-\mathrm{SO}_{3}$ groups and water molecules (Table S2). First, to evaluate the effect of the benzene ring, it is removed from the LAS molecule and the resulting surfactant, hexadecyl sulfonate, is included in an asymmetric system with SLES. $\mathrm{Ca}^{2+}$ adsorbs preferentially at the monolayer of $\mathrm{C}_{16} \mathrm{SO}_{3}$ and the strength of the interaction is comparable to that with LAS. These results show that the presence of the benzene ring in LAS molecules is not crucial for the selective $\mathrm{Ca}^{2+}$ binding. 
(A1)
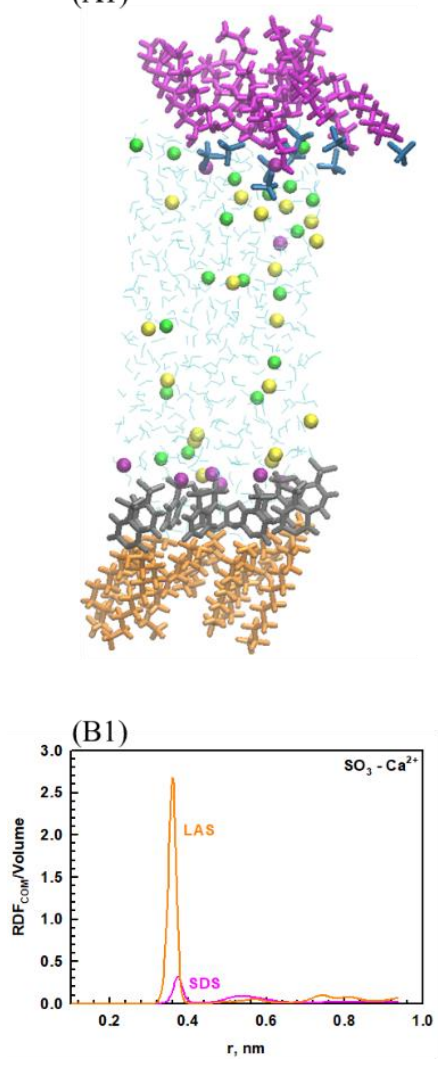

(A2)
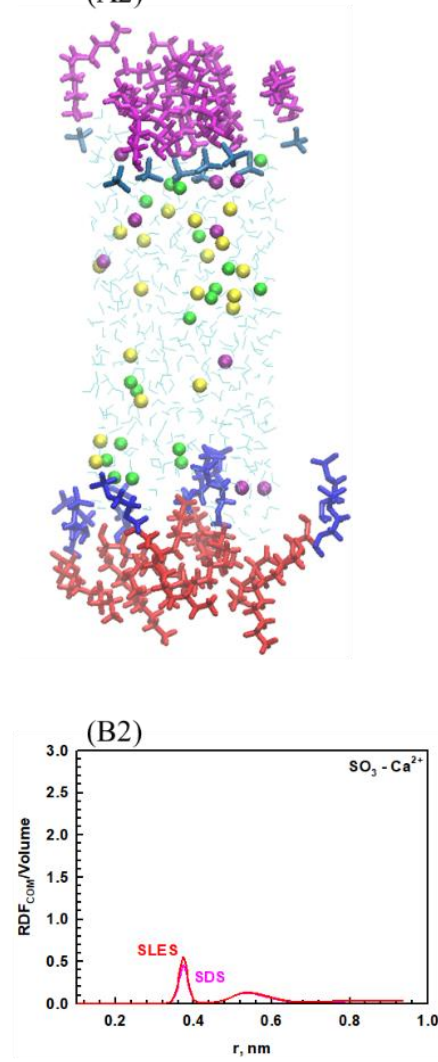

(A3)
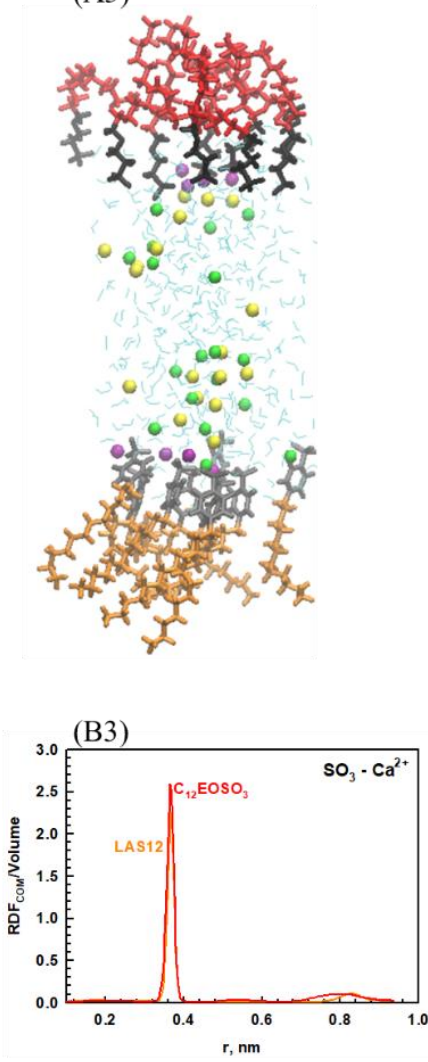

(A4)
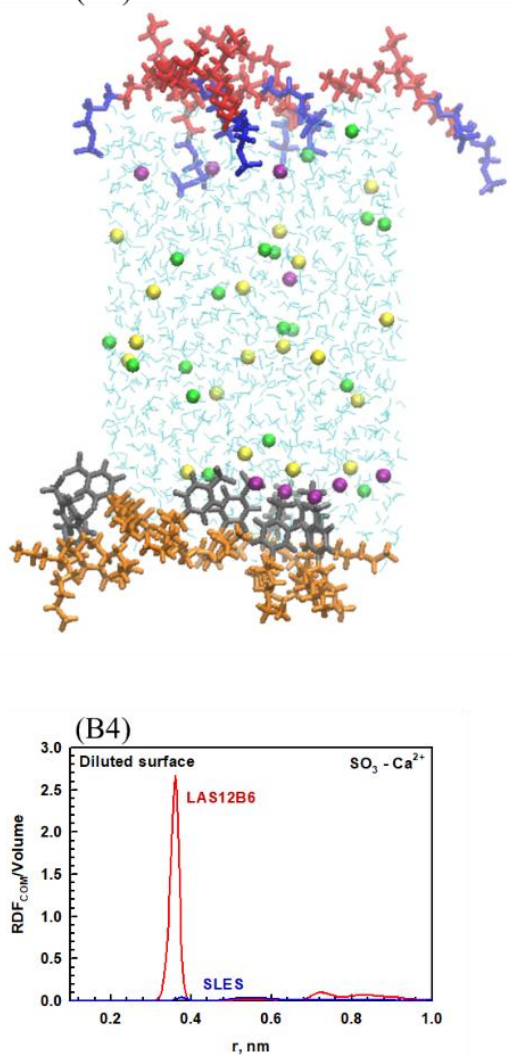

Figure S16. (A) Snapshots of the configurations at $150 \mathrm{~ns}$ of the simulation of the asymmetric concentrated systems (1) SDS/LAS, (2) SDS/SLES, (3) $\mathrm{C}_{12} \mathrm{EOSO}_{3} / \mathrm{LAS} 12$, and asymmetric diluted system of (4) LAS12B6/SLES signed as top/bottom surfaces. (B) RDFs between the $\mathrm{SO}_{3}$ groups in each surfactant and $\mathrm{Ca}^{2+}$.

Table S2. Number of water molecules surrounding $\mathrm{Ca}^{2+}$ ions when the latter are adsorbed on monolayers $\left(\mathrm{N}\left(\mathrm{H}_{2} \mathrm{O}\right) / \mathrm{Ca}^{2+}\right)$, number of hydrogen bonds between $-\mathrm{SO}_{3}$ groups and water molecules $\left(\mathrm{N}_{\mathrm{HB}}\left(\mathrm{SO}_{3}+\mathrm{H}_{2} \mathrm{O}\right)\right)$, and interaction energies between $\mathrm{Ca}^{2+}$ and the surfactants heads $\left(\Delta w\left(\mathrm{SO}_{3}-\mathrm{Ca}^{2+}\right)\right)$ in the systems with model surfactants.

\begin{tabular}{|c|c|c|c|c|}
\hline Asymmetric system & Surfactant & $\mathbf{N}\left(\mathbf{H}_{2} \mathbf{O}\right) / \mathbf{C a}^{2+}$ & $\begin{array}{c}\mathrm{N}_{\mathrm{HB}} \\
\left(\mathrm{SO}_{3}+\mathrm{H}_{2} \mathrm{O}\right)\end{array}$ & $\begin{array}{c}\Delta w\left(\mathrm{SO}_{3}-\mathrm{Ca}^{2+}\right), \\
k T\end{array}$ \\
\hline \multirow[b]{2}{*}{$\mathrm{C}_{16} \mathrm{SO}_{3}+\mathrm{SLES}$} & $\mathrm{C}_{16} \mathrm{SO}_{3}$ & 4 & 3 & 7.8 \\
\hline & SLES & 7 & 6 & 4.0 \\
\hline \multirow{2}{*}{ LAS12 + SLES } & LAS12 & 3 & 2 & 5.5 \\
\hline & SLES & 7 & 5 & 3.7 \\
\hline \multirow{2}{*}{$\mathrm{LAS} 12+\mathrm{C}_{12} \mathrm{EOSO}_{3}$} & LAS12 & 3 & 4 & 5.4 \\
\hline & $\mathrm{C}_{12} \mathrm{EOSO}_{3}$ & 3 & 3 & 5.6 \\
\hline \multirow{2}{*}{ SLES + SDS } & SLES & 6 & 6 & 4.4 \\
\hline & SDS & 7 & 6 & 4.2 \\
\hline \multirow{2}{*}{ LAS $12+$ SDS } & LAS12 & 4 & 3 & 10.2 \\
\hline & SDS & 7 & 6 & 4.1 \\
\hline \multirow{2}{*}{ LAS12B6 + SLES } & LAS12B6 & 4 & 2 & 9.7 \\
\hline & SLES & 8 & 6 & 3.6 \\
\hline
\end{tabular}

The second step involves checking the effect of the hydrophobic chain length. For this purpose, LAS with 12 carbon atoms is used in an asymmetric system with SLES. Again, the result is qualitatively the same as the previous one, namely, $\mathrm{Ca}^{2+}$ binds LAS stronger than 
SLES. The results are illustrated by the average $\mathrm{Ca}^{2+} z$-coordinates (Figure S15B), hydration shells, HB with water, and interaction energies (Table S2). It should be noted that there is some difference in the interaction energies for long- and short-chain LAS - they are higher for C16 LAS. Most probably, this effect is related to the hydration of the hydrophilic head. When it is bound to a hydrophobic chain of different length, the degree of immersion of the surfactant molecule into water is affected. When the hydrophobic part is shorter, the surfactant is more hydrophilic and the interaction with the counterion becomes less preferred and more competitive with hydration.

The third test system is with modified 'SLES' where the sulfate group is replaced by a sulfonate one, bound to the ethoxy group. This molecule is placed in an asymmetric box with LAS12 to maintain the same length of the hydrophobic tail. The simulation of this model showed no selectivity in the binding of the calcium ion to the two surfactants - it is almost the same (Figure S16B3). There is one $\mathrm{Ca}^{2+}$ ion closer to the modified 'SLES' because of the polar ethoxy group. At both monolayers, $\mathrm{Ca}^{2+}$ reduces significantly its hydration shell, resulting in the same number of HBs with water and almost the same interaction energies with both surfactants (Table S2). $\mathrm{Ca}^{2+}$ ions also remain immobilized at the two surfaces (Figure S15C). This signifies the key role of the sulfonate residue for the specific binding of the calcium cations.

The last check was about the effect of the position of the hydrophilic head of LAS along the hydrophobic chain. For this purpose, the most representative isomer of LAS with $\mathrm{C}_{12}$ tail and the benzene ring linked to the sixth carbon atom is simulated in an asymmetric system with SLES as a diluted surface layer. The main results for this system are given in Figure S16 and Table S2. They completely confirm that the key factor for the specific interaction between the LAS and $\mathrm{Ca}^{2+}$ is the sulfonate group, independent of the degree of branching of the molecule. Also with the non-linear LAS much higher interaction energy, smaller number of water molecules surrounding $\mathrm{Ca}^{2+}$, and smaller number of hydrogen bonds between the $\mathrm{SO}_{3}$ group and water are obtained for LAS (LAS12B6) compared to SLES.

The ordering effect of the calcium cations on the LAS molecules is illustrated by the snapshots of the monolayers looked from the bottom (Figure S7A) where very sticky ordering of the $\mathrm{Ca}^{2+}$ between 4 LAS molecules is shown. Thus, aligned rows of surfactants along the entire surface of the layer are formed. In contrast, $\mathrm{Ca}^{2+}$ is randomly ordered between the SLES heads (Figure S7B). These results clearly indicate that the preference of $\mathrm{Ca}^{2+}$ to LAS is mostly due to the sulfonate group. The origin of this strong binding is partially the stronger electrostatic attraction due to the somewhat more negatively charged oxygens in the sulfonate group, when compared to the sulfate group. This is, however, not the sole reason because the difference in the oxygen charges is not that large. This hypothesis is checked by running an additional simulation of the mixed LAS and SLES system using the same charges for the oxygen atoms in the charged heads of the surfactants. The obtained results confirmed the strong and specific interaction between LAS and $\mathrm{Ca}^{2+}$ compared to SLES and $\mathrm{Ca}^{2+}$ by the 
obtained values of the interaction energies, which are $8.1 k T$ and $4.4 k T$ for the $\mathrm{Ca}^{2+}$ interaction with LAS and SLES, respectively.

A second probable factor is the presence of the additional negatively charged oxygen atom in the sulfate group, which needs extra neutralization by cations. The latter seems to be done most efficiently at the SLES monolayers by binding $\mathrm{Na}^{+}$ions, penetrating between the surfactant head-groups. This hypothesis was checked by running another simulation of asymmetric system in which the surfactants were neutralized with calcium ions only, without any sodium ions present. To have an even number of $\mathrm{Ca}^{2+}$ ions which could be bound to both surfactants, one additional molecule of $\mathrm{CaCl}_{2}$ was added. Thus, in total, this model system contained $9 \mathrm{LAS}$ and 9 SLES molecules, $10 \mathrm{Ca}^{2+}$ and $2 \mathrm{Cl}^{-}$. In this case, $5 \mathrm{Ca}^{2+}$ were adsorbed on each monolayer to reach electrostatic equilibrium but the monitoring of the calcium coordinate in $z$-direction indicated much earlier and stronger binding to LAS, compared to SLES. It is illustrated in Figure S14A.

So far, different combinations of molecule groups with the sulfonate group were discussed to clarify the role of the sulfonate group on the $\mathrm{Ca}^{2+}$ binding. It is interesting also to assess the competition between the main molecules (LAS and SLES) and SDS. The latter contains a sulfate group directly bound to the hydrophobic chain. For this purpose, additional simulations of the asymmetric systems LAS+SDS and SLES+SDS in the presence of $\mathrm{Ca}^{2+}$ were conducted. We found that the presence of 1 EO group in the SLES molecule does not affect significantly its interaction with the counterions and water molecules. Almost the same results with respect to the interaction energy with $\mathrm{Ca}^{2+}$ and the hydration of the outer oxygen atoms in the hydrophilic head were obtained. The RDFs are illustrated in Figure S16(A,B) and the number of the water molecules in the $\mathrm{Ca}^{2+}$ hydration shell, the interaction energy between $\mathrm{Ca}^{2+}$ and $-\mathrm{SO}_{3}$, and the number of the hydrogen bonds between $-\mathrm{SO}_{3}$ and the water molecules are presented in Table $\mathbf{S 2}$. 


\section{Influence of the force field: OPLS-AA vs. CHARMM36}

Tang et al. [56] studied preassembled micelles - spherical, short rod-like, periodic cylindrical, or disk-like with different aggregation numbers and at three surfactant concentrations, tuned by changing the size of the model. Three force fields - OPLS-AA, CHARMM36, and GROMOS, were compared. NPT simulations were run for $20 \mathrm{~ns}$ and the last 10 or $5 \mathrm{~ns}$, depending on the aggregation number, were analyzed. All systems were characterized by density profiles of the surfactants and RDFs between the surfactant heads and $\mathrm{Na}^{+}$, between $\mathrm{Na}^{+}$and water molecules, and between sulfur atoms in the surfactant heads.

These authors [56] discuss that, relative to CHARMM36, OPLS-AA predicts larger number of sodium ions condensed within the first counterion shell (up to distance of 0.35 $\mathrm{nm}$ ), resulting in crystal-like cation and (partly) surfactant patches in micelles of aggregation number 100. Such behavior has not been registered experimentally so far. With CHARMM36, on the other hand, no such solidification of the micelles was observed. The authors explained that the 'crystallization' events in OPLS-AA modelled system are probably due to over-binding of the cations. The authors concluded that the force field affects most strongly the surfactant behavior and the interactions with the counterions at micellar aggregation numbers $>100$.

Therefore, we checked the effect of the used force field on our systems. For this purpose, all simulations for the concentrated layers with LAS and SLES were repeated by using CHARMM36, which contains all required parameters for the molecules studied [57]. It is important to emphasize that the charges of the oxygen atoms in LAS and SLES are equal in these simulations, because in CHARMM36, equal empirically-obtained charges are adopted for chemically similar residues (even though they are optimized to reproduce water-residue $a b$ initio interaction energies of isolated clusters), while the ESP-based scheme recommended for OPLS-AA tries to capture the chemical specificity of the particular molecular environment, also taking into account the solvent effect by continuous solvation.

The behavior of the particles is characterized quantitatively by the density profiles (Figures S17 and S18), RDFs (Figure S19), hydration shells of the cations (Figure S20A), and the interaction energies (Figure S20B). Again, all analyses are performed for the last 50 ns of the production part of the simulations.

As explained in the main text, both FFs predict that the $\mathrm{Ca}^{2+}$ ions displace the $\mathrm{Na}^{+}$ions from the adsorption layers by stronger binding to the surfactant heads. However, when the calculations are performed with CHARMM36, almost the same strength of interaction between $\mathrm{Ca}^{2+}$ and the two studied surfactants is obtained. In other words, the simulations with CHARMM36 force field confirm that $\mathrm{Ca}^{2+}$ binds strongly and specifically to LAS, but indicates that strong binding could be present for SLES as well.

Consequently, the observed differences in the RDFs between the surfactants and counterions lead to different hydration shells and interaction energies (Figure S20) compared to those calculated with OPLS-AA (Figures $\mathbf{7}$ and $\mathbf{8}$ of the main text). CHARMM36 yields 
almost the same hydration of a given cation, independent on the surfactant. Only in the absence of $\mathrm{Ca}^{2+}$ in the pure systems, smaller amount of water molecules surrounding $\mathrm{Na}^{+}$than in the other models is obtained. However, in the presence of $\mathrm{Ca}^{2+}$ there is no reduction of the hydration shell of $\mathrm{Na}^{+}$, whereas it loses at least 1 water molecule when the calculations are performed with OPLS-AA (Figure 7B of the main text). On the other hand, the results for $\mathrm{Ca}^{2+}$ show that it loses more than half of the hydration shell, remaining with 2 or 3 water molecules. The big differences reported for the properties of the $\mathrm{Ca}^{2+}$ ions is due to the different protocols used for their parametrization in the two FFs.
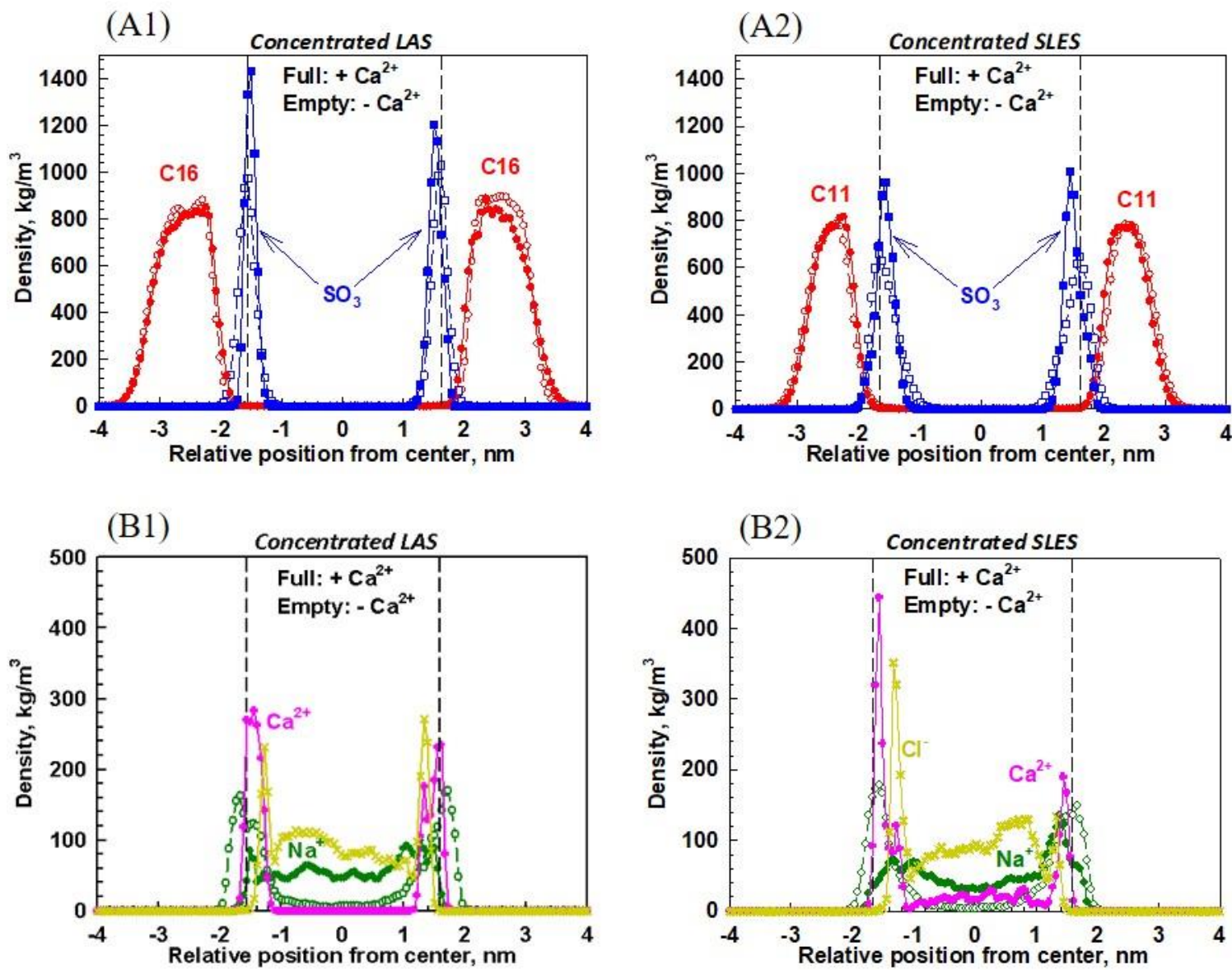

Figure S17. Mass density profiles normal to the interface obtained with CHARMM36 for (A) hydrophobic tails (red circles) and $\mathrm{SO}_{3}$ residues (blue squares) and (B) the inorganic ions green circles for $\mathrm{Na}^{+}$and pink crosses for $\mathrm{Ca}^{2+}$ in the concentrated monolayers of (1) LAS and (2) SLES in the presence (solid line and full symbols) and in the absence (dashed line and empty symbols) of $\mathrm{Ca}^{2+}$. 
(A)

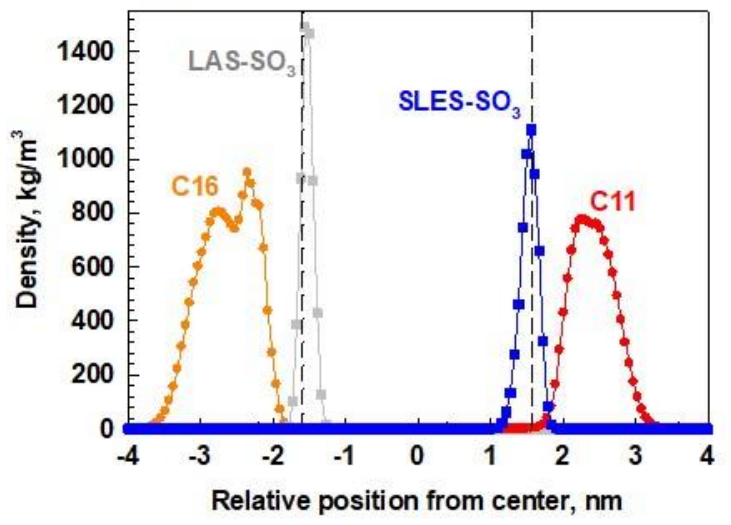

(B)

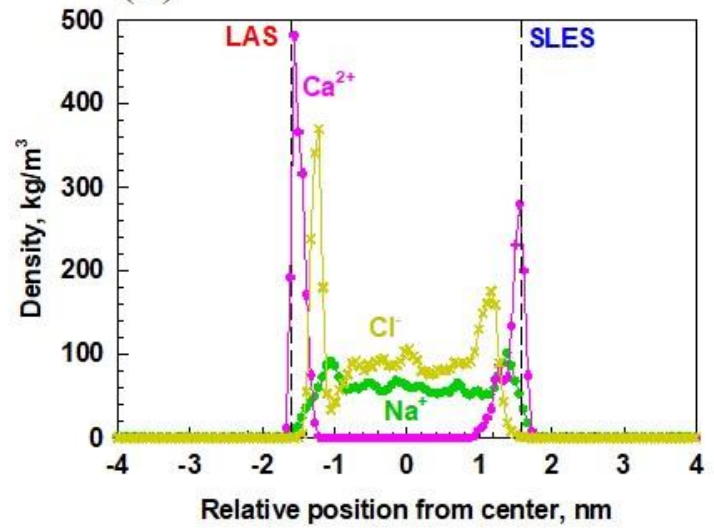

Figure S18. Mass density profiles normal to the interface obtained with CHARMM36 for (A) the hydrocarbon chains and $\mathrm{SO}_{3}$ residues in LAS and SLES, and (B) inorganic ions - green circles for $\mathrm{Na}^{+}$, pink circles for $\mathrm{Ca}^{2+}$, and yellow crosses for $\mathrm{Cl}^{-}$, in the concentrated asymmetric systems.
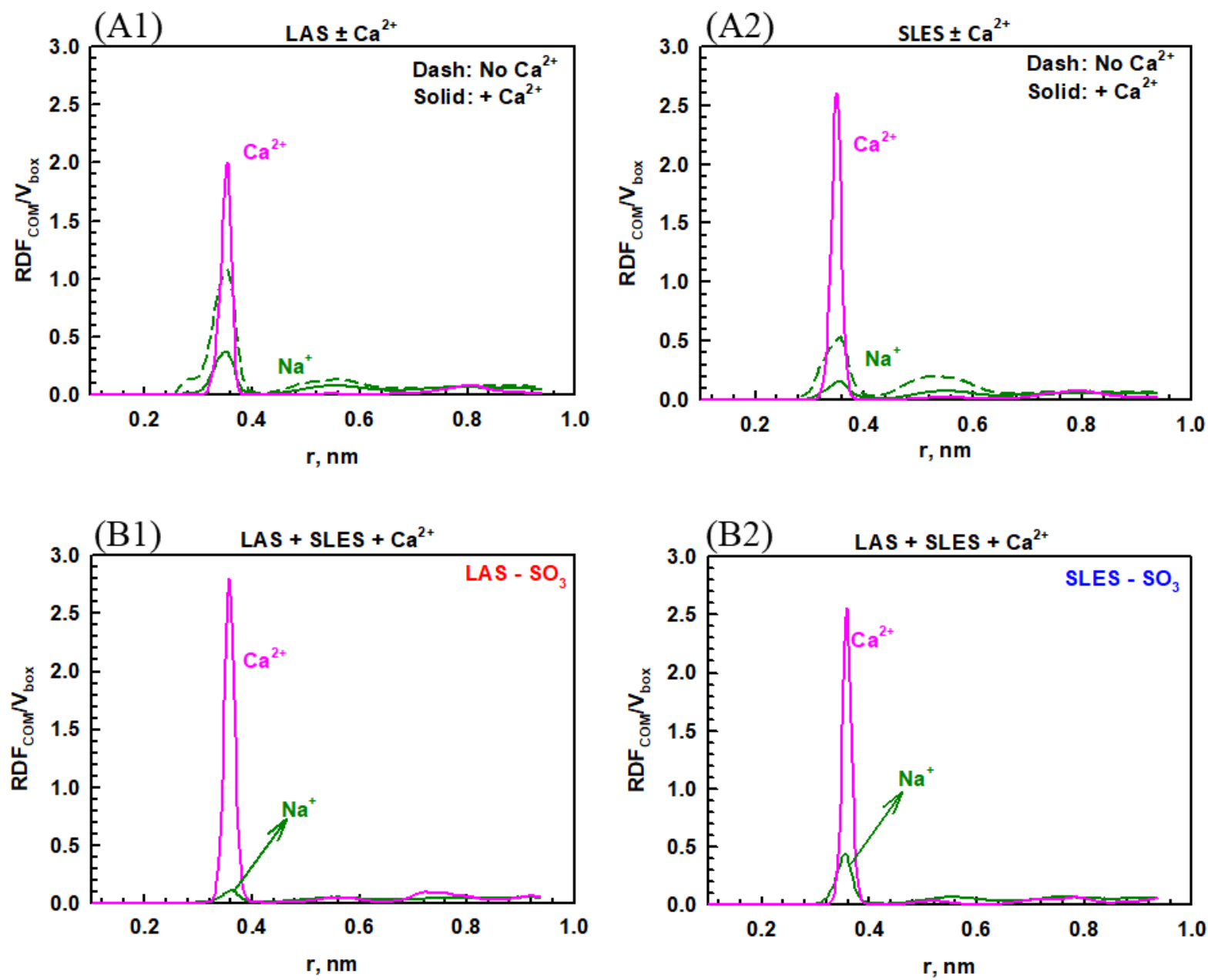

Figure S19. RDFs obtained with CHARMM36 between the cations (green lines $\mathrm{Na}^{+}$, pink lines $\mathrm{Ca}^{2+}$ ) and the $-\mathrm{SO}_{3}$ groups of the surfactants: (1) LAS and (2) SLES in (A) singlesurfactant and (B) asymmetric systems in the presence (solid lines) and in the absence (dashed lines) of $\mathrm{Ca}^{2+}$. 

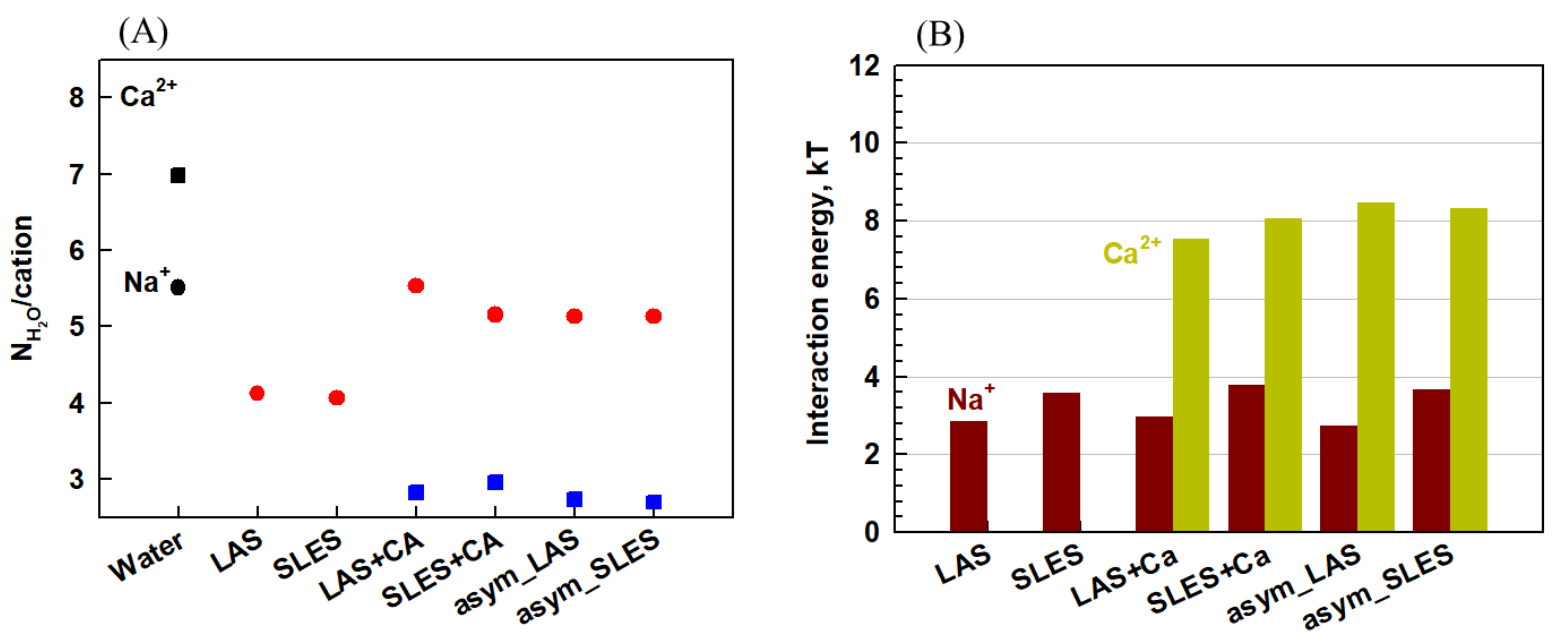

Figure S20. (A) Number of water molecules surrounding the cations $\left(\mathrm{Na}^{+}\right.$: red circles, $\mathrm{Ca}^{2+}$ : blue squares) calculated with CHARMM36. The black symbols are the number of water molecules in bulk solution without any surfactants. (B) Interaction energies between surfactants head groups and counterions $\left(\mathrm{Na}^{+}\right.$: dark red bars, $\mathrm{Ca}^{2+}$ : dark yellow bars) calculated by Equation 3 from the simulations with CHARMM36.

The discrepancy in the results obtained with the two FFs is probably caused by: (1) The different charges of the hydrophilic heads and (2) The Lennard-Jones parameters of the atoms in the surfactants and counterions which are rather different. These differences stem from the unlike philosophies behind the derivation of the two force fields as discussed above.

In our case, the difference of charges may be important, because the equalization of the atomic charges of the oxygen atoms in the sulfate and sulfonate groups leads to significant loss of the specificity of interaction with the calcium cations. The presence of the alkyl chains in surfactants could also affect strongly the charges of differently located oxygen atoms (e.g., the internal and external oxygen atoms in the sulfate group).

The van der Waals parameters are the following for the two force fields: $\varepsilon($ S-OPLS $)=$ $1.046, \varepsilon(\mathrm{S}-\mathrm{CHARMM})=1.996, \varepsilon(\mathrm{O}-\mathrm{OPLS})=0.837, \varepsilon(\mathrm{O}-\mathrm{CHARMM})=0.502, \varepsilon(\mathrm{Na}-\mathrm{OPLS})$ $=0.012, \varepsilon(\mathrm{Na}-\mathrm{CHARMM})=0.196, \varepsilon(\mathrm{Ca}-\mathrm{OPLS})=1.881, \varepsilon(\mathrm{Ca}-\mathrm{CHARMM})=0.502$. These differences come from the fact that the hydration energies of the inorganic ions are the focus in the validation of OPLS-AA both for $\mathrm{Na}^{+}$and $\mathrm{Ca}^{2+}$, whereas in CHARMM36, this target quantity is used only for $\mathrm{Ca}^{2+}$, while the parameters for $\mathrm{Na}^{+}$are tuned primarily to reproduce the interactions with oxygen atoms from lipid head groups (carboxylates, phosphates, and esters) located at the gas-water interface. There is an ad hoc correction term suggested for that [Ref. 57 of the main manuscript]. The results of taking it into account both for LAS and SLES are shown in Figure S21 and reveal that the correction has minor effect. 

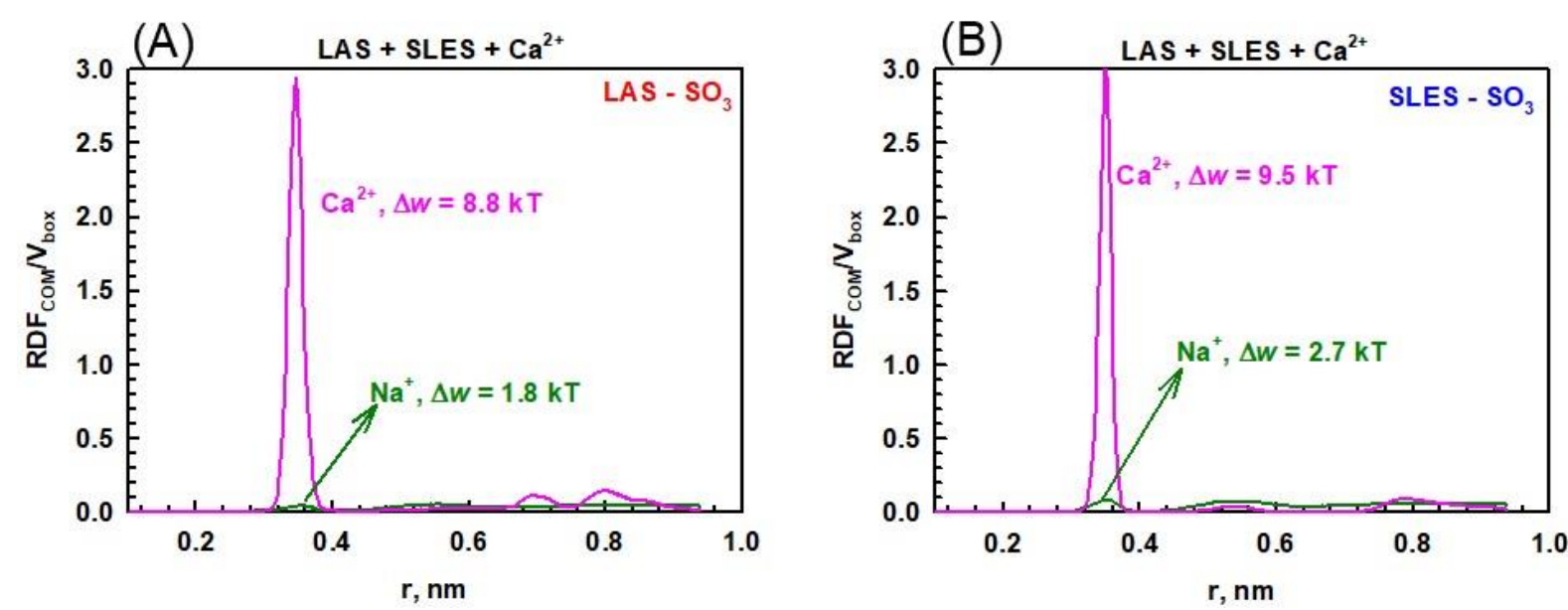

Figure S21. RDFs between the cations (green lines $\mathrm{Na}^{+}$, pink lines $\mathrm{Ca}^{2+}$ ) and the center-ofmass of $\mathrm{SO}_{3}$ groups of (A) LAS and (B) SLES with CHARMM36 with the same atom types on the outer oxygen atoms, which invokes correction terms; the simulations are for the concentrated asymmetric system.

Even though Tang et al. [Ref. 56 of the main manuscript] show that CHARMM36 gives more relevant results for their systems, in our case the outcome obtained with OPLS-AA corresponds much better to the experimentally observed effects than that obtained by CHARMM36. This comparison with the experiment, along with the physical reasoning provided above, allow us to give a preference to the simulations made with OPLS-AA FF for the systems studied in the current article. 